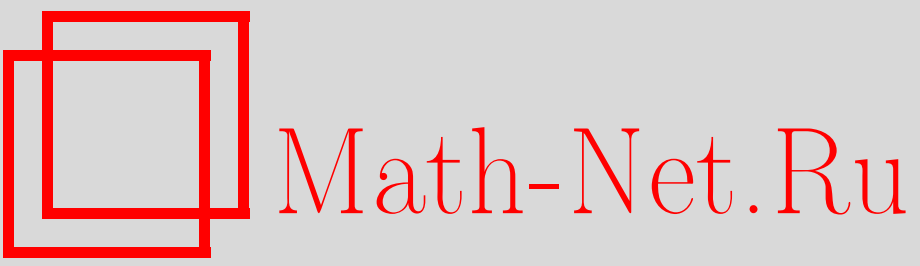

И. Л. Соловцов, Д. В. Ширков, Аналитический подход в квантовой хромодинамике, ТМФ, 1999, том 120, номер 3, 482-510

DOI: https://doi.org/10.4213/tmf792

Использование Общероссийского математического портала Math-Net.Ru подразумевает, что вы прочитали и согласны с пользовательским соглашением

http://www . mathnet.ru/rus/agreement

Параметры загрузки:

IP : 54.157 .27 .8

26 апреля 2023 г., 14:11:21 


\author{
ТЕОРЕТИЧЕСКАЯ \\ И МАТЕМАТИЧЕСКАЯ \\ ФИЗИКА \\ Том 120, № 3 \\ сентябрь, 1999
}

(C) 1999 г.

И. Л. Соловцов*, Д. В. Ширков*

\title{
АНАЛИТИЧЕСКИЙ ПОДХОД В КВАНТОВОЙ ХРОМОДИНАМИКЕ
}

Излагается новая "ренорминвариантная аналитическая формулировка" вычислений в квантовой хромодинамике, в рамках которой ренормгрупповое суммирование коррелируется с аналитичностью по квадрату переданного импульса $Q^{2}$. При этом выражения для инвариантного заряда и матричных элементов модифицируются таким образом, что нефизические особенности типа призрачного полюса не появляются вовсе, будучи по построению скомпенсированными дополнительными непертурбативными вкладами. В рамках новой схемы демонстрируется устойчивость результатов расчетов для ряда физических процессов по отношению к высшим петлевым эффектам и выбору ренормализационного предписания.

Имея в виду применение новой формулировки к характеристикам процессов неупругого лептон-нуклонного рассеяния, мы анализируем структурные функции последнего на основе общих принципов теории, сконцентрированных в интегральном представлении Йоста-Лемана-Дайсона. Используется нестандартная скейлинговая переменная, которая приводит к модифицированным моментам структурных функций, обладающих аналитическими свойствами Челлена-Лемана по переменной $Q^{2}$. Установлена связь этих "модифицированных аналитических моментов" с операторным разложением.

Позаботьтесь о Приниипах и Принципь позаботятся о Вас

Научное творчество Николая Николаевича Боголюбова характеризует сочетание направленности на решение конкретных проблем естествознания с высокой математической культурой. При этом получение физического результата максимально простым образом опирается на наиболее общие принципь теории.

В основе излагаемого ренорминвариантного, аналитического подхода к квантовой хромодинамике, а также его новейших применений лежат работь [1-4], выполненные Боголюбовым совместно со своими ближсайиими учениками. Тесная связь с фундаментальными приниипами квантовой физики является характерной чертой этих исследований.

\section{1. ВВЕДЕНИЕ}

Неотъемлемой частью современной квантовой теории поля (КТП) является предло-

* Лаборатория теоретической физики им. Н. Н. Боголюбова, Объединенный институт ядерных исследований, Дубна, Россия 
женный в середине 50-х годов $[1,2]$ метод ренормализационной группы (РГ). Особенно велика его роль в случаях, когда взаимодействие не является слабым, как, например, в квантовой хромодинамике (КХД). Рассмотрение практически всех адронных процессов на основе КХД немыслимо сегодня вне ренормгруппового анализа. Хорошо известно, что непосредственное решение РГ-уравнения для инвариантного заряда приводит к нефизическим особенностям, например, в однопетлевом приближении - к призрачному полюсу. Учет следующих, многопетлевых поправок не меняет сути дела, а лишь генерирует дополнительные разрезы. Сушествование такого рода особенностей противоречит общим принципам локальной КТП.

Еще в конце 50-х годов Н. Н. Боголюбовым с соавторами [3] был предложен способ разрешения этой проблемы в контексте квантовой электродинамики (КЭД), состояший в объединении метода РГ с требованием аналитичности по переменной $Q^{2}$, вытекающим из отражающего основные принципы локальной КТП [5] известного представления Челлена-Лемана (см. ниже формулу (2.1)).

Инвариантный заряд $\bar{\alpha}\left(Q^{2}\right)$ в КЭД (называемый также “инвариантной или бегушей константой связи" ${ }^{1)}$ ) пропорционален поперечной амплитуде полного фотонного пропагатора, которая удовлетворяет спектральному представлению Челлена-Лемана, отвечающему аналитичности в комплексной плоскости $Q^{2}$ с разрезом вдоль отрииательной части ${ }^{2)}$ действительной оси. Согласно [3] аналитический инвариантный заряд восстанавливается через представление Челлена-Лемана, спектральная плотность в котором определена как мнимая часть инвариантного заряда, найденного методом РГ в евклидовой области и аналитически продолженного в область $\operatorname{Re} Q^{2}<0$. Полученное в [3] явное однопетлевое (и неявное двухпетлевое) выражение для аналитической (константы) связи в КЭД обладает следуюшими важными свойствами:

- призрачный полюс отсутствует;

- как функиия $\alpha$ это выражение в окрестности $\alpha=0$ имеет существенную особенность вида $\exp (-3 \pi / \alpha)$;

- для действительных положительных а допускает разложение по степеням $\alpha$, совпадающее с пертурбативным ;

- имеет конечный ультрафиолетовый (УФ) предел, равный $3 \pi$, который не зависит от әкспериментального значения $\alpha \simeq 1 / 137$.

Применение в работах $[6,7]$ идеи объединения ренорминвариантности и $Q^{2}$-аналитичности для случая КХД обнаружило новые важные свойства аналитической связи. $\mathrm{K}$ их числу относится наличие у $\bar{\alpha}_{\text {ан }}\left(Q^{2}\right)$ инфракрасно стабильной точки, которая оказывается универсальной в том смысле, что ее значение $\alpha=4 \pi / \beta_{0}$ определяется уже однопетлевым вкладом, т.е. не изменяется при учете многопетлевых поправок и, следовательно, является схемно инвариантным. Оно также не зависит от экспериментально определяемого параметра КХД $\Lambda$, а набор кривых $\bar{\alpha}_{\text {ан }}\left(Q^{2} / \Lambda^{2}\right)$, отвечаюших различным значениям $\Lambda$, представляет собой пучок с общей точкой $\bar{\alpha}_{\text {ан }}(0)=4 \pi / \beta_{0}$. Таким образом, аналитический подход существенным образом модифицирует инфракрас-

\footnotetext{
1) Ввиду явной семантической несуразицы последнего термина мы будем использовать выражение инвариантная функция связи или инвариантная связь.

${ }^{2)}$ Используем обозначение $Q^{2}=-q^{2}$, так что евклидовой области отвечают положительные $Q^{2}$.
} 
ное (ИК) поведение пертурбативной инвариантной связи. Мы приведем удобные для применения двухпетлевые приближенные формулы, а также обсудим некоторые феноменологические приложения аналитического подхода ${ }^{3}$.

Настояшую работу можно условно разделить на три части. В первой из них (раздел 2), носяшей характер обзора наших публикаций за последние два года, аналитический инвариантный подход формулируется в целом и подробно излагается применительно к аналитической (константе) связи. Во второй, также обзорной части (раздел 3) формулируется "аналитическая теория возмушений" для физических величин, выраженных через двухточечные объекты типа $D\left(Q^{2}\right)$-функции Адлера, свойства которых могут быть связаны с представлением Челлена-Лемана, и обсуждаются вопросы схемной и петлевой зависимостей.

Наконец, в третьей части (раздел 4) рассматриваются структурные функшии (формфакторы), параметризуюшие сечение неупругого лептон-адронного рассеяния. Для того чтобы связать их с аналитическими функциями по $Q^{2}$, мы исходим из интегрального представления Йоста-Лемана-Дайсона. Опираясь на результаты Боголюбова, Владимирова и Тавхелидзе [4], мы приводим аргументы в пользу введения специальной скейлинговой переменной, моменты структурных функций относительно которой обладают представлением Челлена-Лемана. Вследствие этого к таким моментам можно применить процедуру аналитизации. Рассмотрена также связь аналитических моментов с операторным разложением.

\section{2. ИНВАРИАНТНАЯ АНАЛИТИЧЕСКАЯ ФОРМУЛИРОВКА КХД}

В этом разделе сформулирован метод построения аналитического инвариантного заряда и рассмотрены его основные свойства.

2.1. Ренормгруппа и аналитичность. Сделаем вначале два замечания. Как известно, инвариантный заряд $\bar{\alpha}_{s}\left(Q^{2}\right)$ КХД определяется через произведение пропагаторов и специальной вершинной функции, и возникает вопрос о возможности использования спектрального представления для этого произведения. Этот вопрос был изучен в работе [17], где было показано, что в подобном случае инвариантная связь может быть записана в форме спектрального интеграла. Кроме того, в общем случае эволюция $\bar{\alpha}_{s}\left(Q^{2}\right)$ связана с "бегушим" калибровочным параметром. Здесь мы будем использовать для простоты обшепринятую $\overline{\mathrm{MS}}$-схему, в которой калибровка не оказывает влияния на инвариантный заря д $^{4}$.

Запишем для инвариантной связи $a\left(Q^{2}\right)=\alpha_{s}\left(Q^{2}\right) /(4 \pi)$ спектральное представление

$$
\bar{a}_{\mathrm{aн}}\left(Q^{2}\right)=\frac{1}{\pi} \int_{0}^{\infty} d \sigma \frac{\rho(\sigma, a)}{\sigma+Q^{2}-i \epsilon} .
$$

В ренормгрупповой просуммированной теории возмушений спектральная плотность $\rho(\sigma, a)$ убывает как $1 / \ln ^{2} \sigma$, что позволяет записать спектральное представление без вычитания.

\footnotetext{
3) Развитию и приложениям аналитического подхода посвящены работы [8-16].

4) Аналогичная ситуация имеет место в МОМ-схеме в поперечной калибровке или в МОМ-схеме при использовании специальной ренормировки [18].
} 
В приближении главных логарифмов инвариантная связь имеет вид

$$
\bar{a}^{(1)}\left(Q^{2}\right)=\frac{a}{1+a \beta_{0} \ln \left(Q^{2} / \mu^{2}\right)}=\frac{1}{\beta_{0} \ln \left(Q^{2} / \Lambda^{2}\right)},
$$

где $\beta_{0}=11-2 f / 3$ - однопетлевой коэффициент $\beta$-функции с $f$ активными кварками, $\Lambda=$ $\mu \exp \left[-1 / 2 a_{\mu} \beta_{0}\right]-$ параметр шкалы КХД. Соответствуюшая спектральная плотность имеет вид

$$
\frac{a^{2} \beta_{0} \pi}{\left[1+a \beta_{0} \ln \left(\sigma / \mu^{2}\right)\right]^{2}+\left[a \beta_{0} \pi\right]^{2}}=\frac{1}{\beta_{0}} \frac{\pi}{\ln ^{2}\left(\sigma^{2} / \Lambda^{2}\right)+\pi^{2}}=\rho^{(1)}(\sigma, a),
$$

а ее подстановка в спектральный интеграл (2.1) дает однопетлевую аналитическую функцию связи

$$
\bar{a}_{\mathrm{aH}}^{(1)}\left(Q^{2} / \Lambda^{2}\right)=\frac{1}{\beta_{0}}\left[\frac{1}{\ln \left(Q^{2} / \Lambda^{2}\right)}+\frac{\Lambda^{2}}{\Lambda^{2}-Q^{2}}\right] .
$$

Первое слагаемое в правой части сохраняет обычное УФ-поведение инвариантной связи. Второе слагаемое, которое появляется из спектрального представления и обеспечивает правильные аналитические свойства, компенсируя призрачный полюс при $Q^{2}=\Lambda^{2}$, является сушественно непертурбативным (см. обшее обсуждение этого вопроса в работе [19]). При разложении в ряд Тейлора это слагаемое вклада не дает. Таким образом, принципы причинности и спектральности, выраженные в форме $Q^{2}$-аналитичности, сигнализируют о том, что дело не исчерпывается теорией возмущений. Требование выполнения правильных аналитических свойств приводит к появлению степенных по $Q^{2}$ вкладов, не видимых в исходном пертурбативном разложении. Отметим также, что, в отличие от электродинамики, в КХД благодаря свойству асимптотической свободы проявление таких непертурбативных вкладов в эффективной функции связи имеет место не при фантастически высоких энергиях, а в области низких энергий и передач импульса, достижимых в реальных экспериментах.

Таким образом, синтез ренормгрупповой инвариантности и аналитичности приводит к аналитическому инвариантному заряду без логарифмического полюса и с конечным ИК-значением ${ }^{5)} \bar{\alpha}_{\text {ан }}(0)=4 \pi / \beta_{0} \simeq 1.396$. Это предельное значение не зависит от экспериментальной информации, связанной с точкой нормировки $a=a\left(\mu^{2}\right)$ или параметром $\Lambda$, а определяется лишь коэффициентом $\beta$-функции, связанным с общей групповой структурой лагранжиана. На рис. 1 изображен пучок кривых $\bar{\alpha}_{\text {ан }}\left(Q^{2}\right)$, отвечающих различным значениям $\Lambda$, а также соответствующие тем же $\Lambda$ обычные решения.

График однопетлевой $\beta$-функции, иллюстрируюший наличие инфракрасно стабильной точки в аналитическом подходе, изображен на рис. 2. По горизонтальной оси отложен параметр $\beta_{0} a$, по вертикальной оси функция $-\beta(a)$. Отметим, что в однопетлевом случае имеет место симметрия относительно точки $\beta_{0} a=1 / 2$, которая разрушается при учете высших порядков.

Перейдем к двухпетлевому случаю. Соответствующая $\beta$-функция имеет вид

$$
\beta(a)=-\beta_{0} a^{2}\left(1+b_{1} a\right), \quad b_{1}=\frac{\beta_{1}}{\beta_{0}}, \quad \beta_{1}=102-\frac{38 f}{3}=64 .
$$

\footnotetext{
5) Для численных оценок при малых $Q^{2}$ мы используем число активных кварков $f=3$.
} 


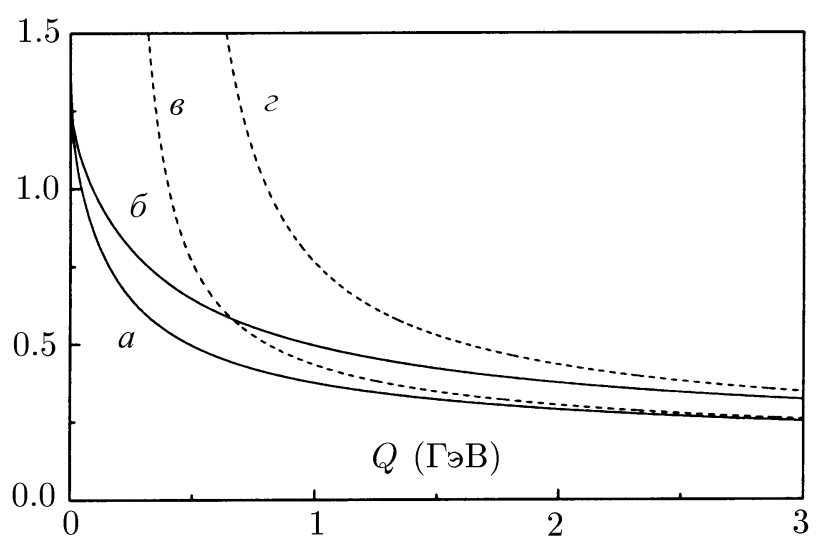

Рис.1. Поведение однопетлевой аналитической константы связи $\bar{\alpha}_{\text {ан }}\left(Q^{2}\right): a-$ для $\Lambda=$ $200 \mathrm{MэB}, 6$ - для $\Lambda=400$ МэВ. Кривые $в$ и г соответствуют теории возмущений для тех же значений параметра $\Lambda$.

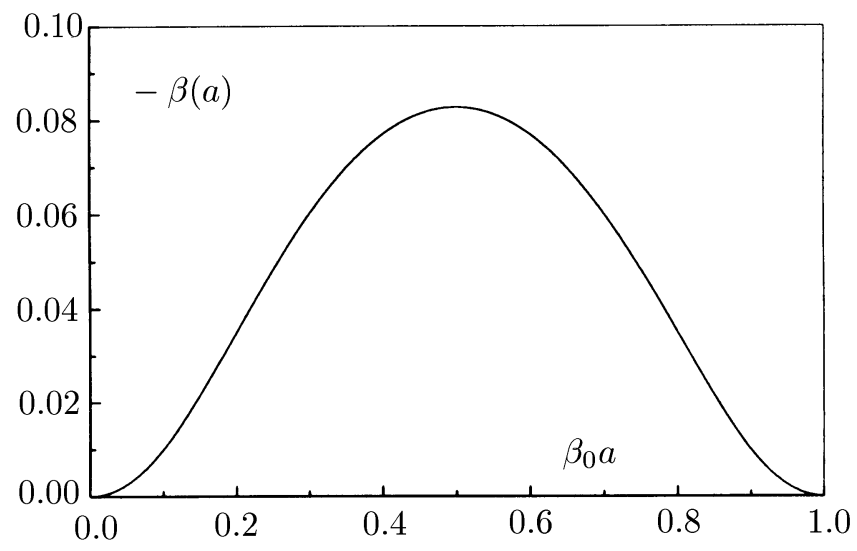

Рис. 2. График однопетлевой $\beta$-функции.

Интегрирование ренормгруппового уравнения дает трансцендентное соотношение

$$
\beta_{0} \ln x=\frac{1}{\bar{a}(x)}-b_{1} \ln \left(1+\frac{1}{b_{1} \bar{a}(x)}\right),
$$

которое может быть разрешено в терминах функции Ламберта [20, 21].

Полученная из этого уравнения спектральная плотность изображена на рис. 3 (кривая б). Она оказывается очень близкой к спектральной плотности, соответствуюшей явному итеративному решению уравнения (2.6)

$$
\bar{a}^{(2)}\left(Q^{2}\right)=\frac{1}{\beta_{0} \ell+b_{1} \ln \left(1+\beta_{0} \ell / b_{1}\right)}, \quad \ell=\ln \frac{Q^{2}}{\Lambda^{2}},
$$




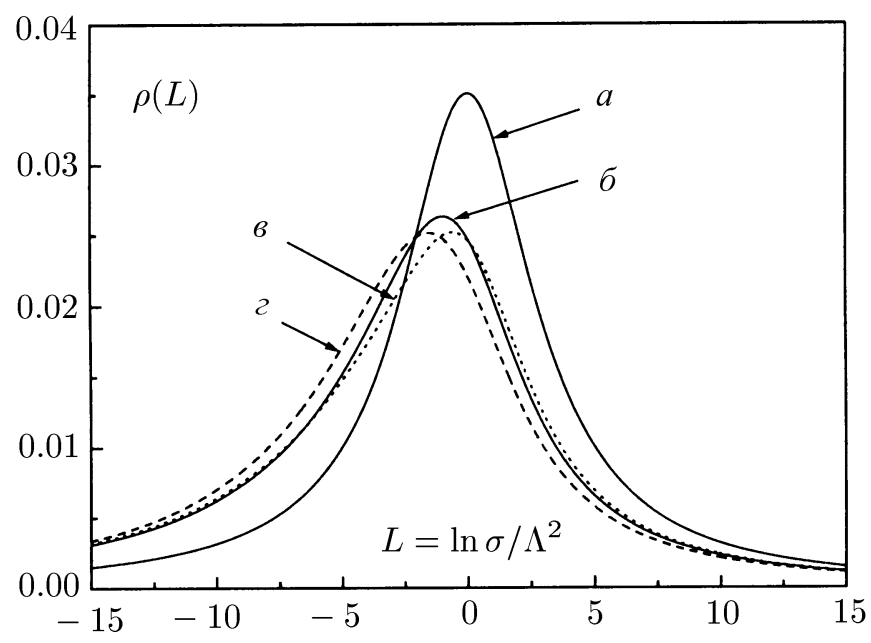

Рис. 3. Спектральные плотности: $a$ - точная однопетлевая, 6 - точная двухпетлевая, $в$ приближенное двухпетлевое выражение (итеративное решение), г- точная трехпетлевая функция.

удобному для последуюшего анализа.

Решению (2.7) отвечает спектральная функция

$$
\begin{aligned}
& \beta_{0} \rho^{(2)}(\sigma)=\frac{I(L)}{R^{2}(L)+I^{2}(L)}, \quad L=\ln \frac{\sigma}{\Lambda^{2}} \\
& R(L)=L+B_{1} \ln \sqrt{\left(1+\frac{L}{B_{1}}\right)^{2}+\left(\frac{\pi}{B_{1}}\right)^{2}}, \\
& I(L)=\pi+B_{1} \arccos \frac{B_{1}+L}{\sqrt{\left(B_{1}+L\right)^{2}+\pi^{2}}}, \quad B_{1}=\frac{\beta_{1}}{\beta_{0}^{2}} .
\end{aligned}
$$

Ее график изображен на рис. 3 (кривая $в$ ), где мы также привели однопетлевой (кривая $а$ ) и трехпетлевой (кривая 2) результаты. Трехпетлевая $\rho^{(3)}$, изображенная на рис. 3, получена в $\overline{\mathrm{MS}}$-схеме из точного интеграла РГ-уравнения с трехпетлевым коэффициентом

$$
\beta_{2}=\frac{2857}{2}-\frac{5033}{18} f+\frac{325}{54} f^{2} \stackrel{f=3}{=} \frac{3863}{6} \simeq 643,83
$$

Как видно из рис. 3 , начиная с двухпетлевого уровня наступает стабилизация в поведении спектральных плотностей, причем, как будет показано ниже, площади под всеми этими кривыми совпадают, что и соответствует универсальности $\bar{a}_{\text {ан }}(0)$.

Для того чтобы получить $\bar{a}_{\text {ан }}^{(2)}\left(Q^{2}\right)$, следует подставить спектральную плотность $(2.8)$ в (2.1). Получаемый интеграл не вычисляется явно ${ }^{6}$. . Восстановление правильных аналитических свойств происходит не только устранением полюса, но и за счет выгитания

\footnotetext{
${ }^{6)}$ Ниже мы приведем явные приближенные формулы для этого случая.
} 


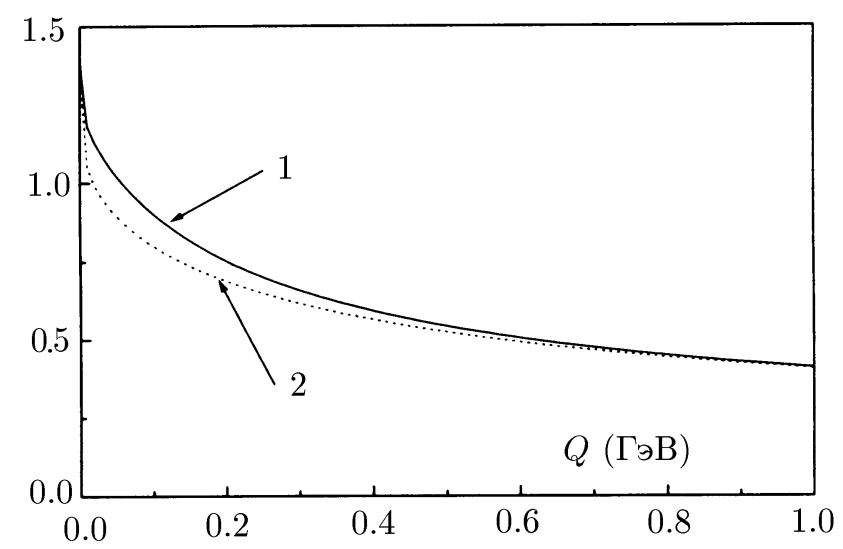

Рис.4. Стабильность аналитического инвариантного заряда по отношению к высшим петлевым поправкам. Использована нормировка на массе $\tau$-лептона $\bar{\alpha}_{\text {ан }}\left(M_{\tau}^{2}\right)=0.34$ для $f=3 ; 1$ - однопетлевое приближение, 2 - двух- и трехпетлевое приближения.

нефизического разреза $0<Q^{2}<\Lambda^{2} \exp \left(-B_{1}\right)$, обусловленного двойной логарифмической зависимостью в $(2.7)$.

Результат численных расчетов для $f=3$ при нормировке на точку $\bar{\alpha}_{\text {ан }}\left(M_{\tau}^{2}\right)=0.34$ приведен на рис. 4 вместе с однопетлевой кривой (соответствуюшие значения $\Lambda$ даны в табл. 1). Трехпетлевая $\overline{\mathrm{MS}}$-кривая практически совпадает с двухпетлевой с точностью порядка $1 \%$. Таким образом, в отличие от теории возмущений аналитичность приводит к существенной стабилизации поведения инвариантного заряда в ИК-области. С учетом свойства асимптотической свободы мы получаем стабильность во всей евклидовой области $0<Q^{2}<\infty$.

Отметим здесь, что универсальность поведения аналитической функции связи не является следствием использования конкретной двухпетлевой формулы (2.7). В частности, тот же вывод остается справедливым и при использовании точного решения (2.6). Таким образом, ИК-стабильность аналитического заряда является внутренним свойством метода и обеспечивается неаналитическими по $\alpha_{s}$ вкладами. Обсуждаемый подход не привносит каких-либо дополнительных параметров в теорию, а оперирует лишь с параметром шкалы $\Lambda$ или с некоторой точкой нормировки.

2.2. Вычитание нефизических особенностей. Аналитическое выражение для инвариантной связи получено при использовании спектрального представления (2.1), которое гарантирует правильные аналитические свойства в комплексной $Q^{2}$-плоскости и эффективно сводится к вычитанию нефизических особенностей (полюса и разрезов). Удобно выделить эти члены явно.

Рассмотрим комплексную плоскость переменной $z=Q^{2} / \Lambda^{2}$. Метод вычитания сингулярностей позволяет получить явное выражение для аналитической связи в однопетлевом случае. Действительно, выражение $\beta_{0} \bar{a}^{(1)}(z)=1 / \ln z$ имеет нефизический полюс при $z=1$ с вычетом $\operatorname{res}\left[\beta_{0} \bar{a}^{(1)}(z), z=1\right]=1$, устранение которого сводится к добавлению слагаемого $1 /(1-z)$, так что выражение с правильными аналитическими свойствами имеет вид (2.4). 


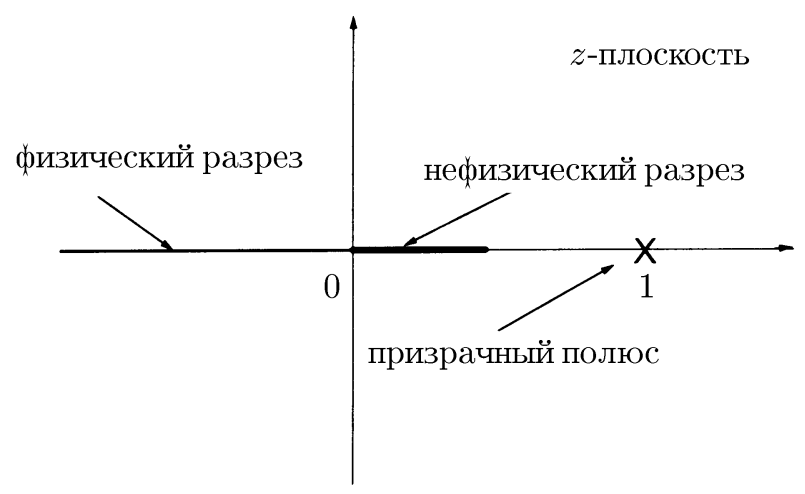

Рис. 5. Двухпетлевые особенности в комплексной плоскости $\left(z=Q^{2} / \Lambda^{2}\right)$.

В двухпетлевом случае рассмотрим вначале выражение (2.7), которое помимо призрачного полюса при $z=1$ с вычетом $\operatorname{res}\left[\beta_{0} \bar{a}^{(2)}(z), z=1\right]=1 / 2$ имеет нефизический разрез вдоль положительной части вешественной оси $0<z<\exp \left(-B_{1}\right)$ (см. рис.5). Вьгитание осушествляется полюсным слагаемым

$$
\beta_{0} \Delta \bar{a}_{\text {pole }}^{(2)}(z)=\frac{1}{2} \frac{1}{1-z}
$$

и интегралом, удаляюшим нефизический разрез,

$$
\beta_{0} \Delta \bar{a}_{\mathrm{cut}}^{(2)}(z)=\frac{1}{\pi} \int_{0}^{\exp \left(-B_{1}\right)} \frac{d \sigma}{\sigma-z} \frac{\pi B_{1}}{\left[\ln (\sigma)+B_{1} \ln \left(-1-\ln (\sigma) / B_{1}\right)\right]^{2}+\pi^{2} B_{1}^{2}} .
$$

В результате аналитический инвариантный заряд записывается в виде

$$
\beta_{0} \bar{a}_{\mathrm{aH}}^{(2)}(z)=\beta_{0} \bar{a}^{(2)}(z)+\beta_{0} \Delta \bar{a}_{\mathrm{pole}}^{(2)}(z)+\beta_{0} \Delta \bar{a}_{\mathrm{cut}}^{(2)}(z) .
$$

Удобство этой записи состоит в том, что аналитическая связь представляется в виде суммы обычного выражения и дополнительных членов, имеюших непертурбативную природу. Их вклад может быть представлен в виде разложения по степеням параметра $\Lambda^{2} / Q^{2}$ (см. ниже $\left.(2.22)\right)$.

2.3. Универсальность $\bar{a}_{\text {ан }}(0)$. Универсальное значение при $Q^{2}=0$ в этом случае складьвается из вклада полюсного слагаемого $\Delta \bar{a}_{\text {pole }}^{(2)}(z=0)=1 /\left(2 \beta_{0}\right)$ и вклада слагаемого (2.11), который может быть представлен в виде

$$
\Delta \bar{a}_{\mathrm{cut}}^{(2)}(z=0)=\frac{1}{\beta_{0}} \int_{0}^{\infty} \frac{d x}{(x+1-\ln x)^{2}+\pi^{2}}=\frac{1}{2 \beta_{0}} .
$$

Суммарный вклад приводит к универсальному выражению $\bar{a}_{\text {ан }}(0)=1 / \beta_{0}$.

В рассмотренном выше способе аппроксимации исходной двухпетлевой константы вычет в полюсе - главной нефизической особенности - не зависит от двухпетлевого коэффициента $\beta$-функции и может показаться, что именно этот факт и приводит к независимости $\bar{a}_{\text {ан }}(0)$ от высших петлевых поправок. Однако, как мы отмечали выше, причина 
универсальности $\bar{a}_{\text {ан }}(0)$ иная и не сводится к выбору конкретного способа аппроксимации исходной инвариантной связи. Поясним это. Обычное асимптотическое двухпетлевое выражение может быть получено при разложении функции

$$
\beta_{0} \bar{a}^{(2)}(z)=\frac{1}{\ln z+B_{1} \ln \left(1+\ln \frac{z}{C}\right)},
$$

где $C$ - некоторая константа. Выражение (2.13) правильно воспроизводит обычный, не зависяший от введенной константы $C$ УФ-предел

$$
\bar{\alpha}_{s}=\frac{4 \pi}{\beta_{0}}\left[\frac{1}{\ln \left(Q^{2} / \Lambda^{2}\right)}-\frac{\beta_{1}}{\beta_{0}^{2}} \frac{\ln \ln \left(Q^{2} / \Lambda^{2}\right)}{\ln ^{2}\left(Q^{2} / \Lambda^{2}\right)}\right] .
$$

Вместе с тем вычет в полюсе теперь зависит от двухпетлевого коэффициента $\beta$-функции через $B_{1}$ :

$$
\operatorname{res}\left[\beta_{0} \bar{a}^{(1)}(z), z=1\right]=\frac{1}{1+B_{1} / C}
$$

и, следовательно, эту же зависимость будет содержать соответствующий компенсирующий член

$$
\beta_{0} \Delta \bar{a}_{\text {pole }}^{(2)}(z)=\frac{1}{1+B_{1} / C} \frac{1}{1-z}
$$

с вкладом в $\bar{a}_{\text {ан }}(0)$, равным

$$
\Delta \bar{a}_{\text {pole }}^{(2)}(0)=\frac{1}{\beta_{0}} \frac{1}{1+B_{1} / C} .
$$

Вклад в $\bar{a}_{\text {ан }}(0)$ слагаемого, компенсирующего нефизический разрез, теперь определяется интегралом

$$
\Delta \bar{a}_{\mathrm{cut}}^{(2)}(z=0)=\frac{1}{\beta_{0}} \frac{C}{B_{1}} \int_{0}^{\infty} \frac{d x}{\left[(x+1) C / B_{1}-\ln x\right]^{2}+\pi^{2}}=\frac{B_{1}}{\beta_{0}\left(B_{1}+C\right)},
$$

что вместе с полюсным вкладом (2.17) дает не зависяшее ни от $C$, ни от $B_{1}$ универсальное значение $\bar{a}_{\text {ан }}(0)=1 / \beta_{0}$.

При учете вклада высших петель для доказательства универсальности ИК-предела в аналитическом подходе оказывается удобным использовать рассмотрение в терминах комплексной величины $\zeta=1 / a$. Здесь мы приведем более простые аргументы, основываясь на разложении пертурбативного заряда в двойной ряд по степеням $\ln ^{m}\left(\ell / \ell^{k}\right)$. Для $\bar{a}_{\text {ан }}(0)$ в этом случае можно записать

$$
\bar{a}_{\mathrm{aн}}(0)=\frac{1}{\pi} \int_{-\infty}^{\infty} d L \varrho(L)=\frac{1}{\beta_{0}}+\sum_{k=1}^{\infty} \sum_{m=0}^{k} \alpha_{k, m} \Delta \bar{a}_{k, m}(0),
$$

где вклад от высших петель определяется выражением

$$
\Delta \bar{a}_{k, m}(0)=\frac{1}{\pi} \operatorname{Im} \int_{-\infty}^{\infty} d L \frac{\ln ^{m}(L-i \pi)}{(L-i \pi)^{k+1}} .
$$

Отсутствие сингулярностей у подынтегрального выражения в (2.20) в нижней полуплоскости немедленно дает $\Delta \bar{a}_{k, m}(0)=0$, что и доказывает универсальность значения инфракрасно стабильной точки аналитического заряда. 
Таким образом, требование аналитичности бегущего заряда сушественным образом модифицирует теорию возмушений в ИК-области. Определяюшим здесь является универсальность ИК-предельного значения аналитической функции связи - инвариантность по отношению к высшим петлевым поправкам, которая приводит к тому, что семейство кривых инвариантного заряда, соответствуюших различным петлевым аппроксимациям, выглядит как пучок с обшей точкой при $Q^{2}=0$. Кроме того, в УФ-области эти кривые, естественно, сближаются в силу свойства асимптотической свободы. В рассматриваемом подходе, в отличие от стандартной теории возмущений, возникает исключительно стабильная картина поведения инвариантного заряда по отношению к высшим поправкам. Факт стабильности важен для феноменологических применений, когда затрагивается интервал энергий порядка или меньше нескольких ГэВ.

2.4. Приближенные формулы. Явная однопетлевая формула (2.4) весьма проста, и ее использование не вызывает каких-либо затруднений. В двухпетлевом случае аналитическая константа записывается в виде интегрального представления и представляет интерес найти приближенные явные, удобные для применений выражения.

Здесь мы рассмотрим две такие формулы. Первое выражение непосредственно следует из картины вычитания нефизических особенностей, изложенной в третьем разделе работы. В соответствии с ней аналитическая связь представима в виде

$$
\bar{\alpha}_{\mathrm{aн}}\left(Q^{2}\right)=\bar{\alpha}_{\mathrm{TB}}\left(Q^{2}\right)+\Delta \bar{\alpha}_{\text {sing }}\left(Q^{2}\right),
$$

где $\bar{\alpha}_{\text {Тв }}\left(Q^{2}\right)$ - пертурбативный вклад, а член $\Delta \bar{\alpha}_{\text {sing }}\left(Q^{2}\right)$ вычитает нефизические особенности. Для пертурбативного слагаемого, взятого в виде $(2.7)$, член, удаляюший нефизические особенности, представим в виде двух слагаемых, вычитающих соответственно нефизические полюс и разрез. Слагаемое, компенсирующее полюс, имеет простой вид. Для компенсирующего разрез слагаемого воспользуемся тем, что коэффициенты разложения $C_{k}$,

$$
\Delta \bar{\alpha}_{\mathrm{cut}}\left(Q^{2}\right)=-\frac{4 \pi}{\beta_{0}} \sum_{k=1}^{\infty}\left(\frac{\Lambda^{2}}{Q^{2}}\right)^{k} C_{k}, \quad C_{k}=\int_{0}^{\infty} d t \frac{\exp \left[-B_{1} k(t+1)\right]}{(t+1-\ln t)^{2}+\pi^{2}}
$$

численно малы и быстро убывают $\left(C_{1}=0.0354, C_{2}=0.0079, C_{3}=0.0023, \ldots\right)$. Учитьвая в разложении лишь первый член, получаем простую интерполяционную формулу

$$
\begin{aligned}
\bar{\alpha}_{\text {aппр }}^{(2)}\left(Q^{2}\right)= & \frac{4 \pi}{\beta_{0}}\left\{\frac{1}{\ln \left(Q^{2} / \Lambda^{2}\right)+B_{1} \ln \left[1+\ln \left(Q^{2} / \Lambda^{2}\right) / B_{1}\right]}-\right. \\
& \left.-\frac{1}{2} \frac{\Lambda^{2}}{Q^{2}-\Lambda^{2}}-\frac{\Lambda^{2}}{Q^{2}} C_{1}\right\}
\end{aligned}
$$

хорошо аппроксимирующую ${ }^{7)}$ двухпетлевую аналитическую связь при умеренно больших $Q^{2}$. На интервале $1<Q<1.5$ ГэВ точность приближения не хуже $0.4 \%$, а при больших значениях $Q$ различие формул становится пренебрежимо малым. Таким образом, выражение (2.23) вполне приемлемо в области умеренно больших $Q \geqslant 1$ ГэВ.

\footnotetext{
7) Приближенная формула для двухпетлевой поправки $\left[\bar{a}^{2}\right]$ ан к физическим величинам типа $D$-функции также может быть найдена этим способом.
} 
ТАБлицА 1. Зависимость пертурбативного и аналитического одно- и двухпетлевого значений масштабного параметра (в МэВ) для $f=3$ от точки нормировки $\bar{\alpha}_{s}\left(M_{\tau}^{2}\right)$.

\begin{tabular}{|c|c|c|c|c|c|}
\hline $\bar{\alpha}_{s}\left(M_{\tau}^{2}\right)$ & 0.30 & 0.32 & 0.34 & 0.36 & 0.38 \\
\hline$\Lambda_{\text {TВ }}^{(1)}$ & 173 & 201 & 228 & 256 & 283 \\
\hline$\Lambda_{\text {aн }}^{(1)}$ & 197 & 235 & 275 & 319 & 366 \\
\hline$\Lambda_{\text {TВ }}^{(2)}$ & 333 & 377 & 419 & 460 & 500 \\
\hline$\Lambda_{\text {ан }}^{(2)}$ & 434 & 516 & 607 & 706 & 814 \\
\hline$\Lambda_{\text {аппр }}^{(2)}$ & 423 & 500 & 582 & 671 & 777 \\
\hline
\end{tabular}

Однако в ряде случаев возникает необходимость иметь дело с меньшими значениями $Q$ вплоть до $Q \simeq 0$. Для такого рода задач формула (2.23) становится не применимой из-за плохой аппроксимации компенсируюшего разрез члена степенным разложением (2.22). Использование следуюшей приближенной формулы для двухпетлевого аналитического заряда:

$$
\begin{gathered}
\bar{\alpha}_{\text {aппр }}^{(2)}\left(Q^{2}\right)=\frac{4 \pi}{\beta_{0}}\left[\frac{1}{\ell_{2}\left(Q^{2}\right)}+\frac{1}{1-\exp \left[\ell_{2}\left(Q^{2}\right)\right]}\right], \\
\ell_{2}\left(Q^{2}\right)=\ln \frac{Q^{2}}{\Lambda^{2}}+B_{1} \ln \sqrt{\ln ^{2} \frac{Q^{2}}{\Lambda^{2}}+4 \pi^{2}},
\end{gathered}
$$

возможно и при $Q \simeq 0$. Формула (2.24) воспроизводит УФ двухпетлевое асимптотическое поведение (2.14) и универсальное предельное значение при $Q^{2}=0$. Это выражение аппроксимирует точное при $Q \geqslant 1$ ГэВ в пределах $1 \%$ и может использоваться при всех $Q^{2}$.

В аналитической функции связи при достаточно больших $Q^{2}$ доминирует ее пертурбативная составляющая. Однако уже при $Q=M_{\tau}$ непертурбативный вклад оказывается существенным. Сравнение значений параметра $\Lambda$, соответствующих пертурбативному и аналитическому подходам, демонстрируется в табл. 1. Результат, полученный по формуле (2.23), с высокой точностью воспроизводит точный двухпетлевой расчет и здесь не приводится. Для двухпетлевой пертурбативной формулы использовано выражение (2.7), наиболее подходящее для нашего анализа ${ }^{8)}$. Последняя строка соответствует приближенному выражению (2.24).

\section{3. АНАЛИТИЧЕСКАЯ ТЕОРИЯ ВОЗМУЩЕНИЙ}

В этом разделе дан краткий обзор применений аналитического подхода к анализу некоторых процессов. Для рассматриваемых здесь физических величин будем использовать процедуру аналитизации всего пертурбативного выражения, содержашего высшие

\footnotetext{
8) Отметим, что использование формулы (2.7) в качестве пертурбативной приводит к несколько бо́льшим значениям $\Lambda$, чем при обработке по формуле (2.14).
} 
ТАБлицА 2. Инфракрасная интегральная характеристика $\bar{\alpha}_{s}\left(k^{2}\right)$, вычисленная в одно- и двухпетлевом приближениях, при нормировке на массе $\tau$-лептона.

\begin{tabular}{|c|c|c|c|}
\hline $\bar{\alpha}_{\text {ан }}\left(M_{\tau}^{2}\right)$ & 0.34 & 0.36 & 0.38 \\
\hline$A_{1 \text { петля }}(2$ ГэВ $)$ & 0.50 & 0.52 & 0.55 \\
\hline$A_{2 \text { петли }}(2$ ГэВ $)$ & 0.48 & 0.50 & 0.52 \\
\hline
\end{tabular}

степени инвариантного заряда [9]. Такой способ действий приводит к так называемой аналитической теории возмущений (АТВ).

Рассмотрена интегральная характеристика инвариантного заряда в ИК-области, информация о которой извлекается из физики струй, а также процессы $e^{+} e^{-}$-аннигиляции в адроны и инклюзивный распад $\tau$-лептона. На этом материале изучается проблема зависимости теоретических результатов от выбора схемы перенормировки. Показано, что применение АТВ позволяет заметно снизить схемную зависимость. В свою очередь, это означает, что достигнутый для многих процессов трехпетлевой уровень оказывается практически не зависимым от выбора схемы.

3.1. Интегральная характеристика $\bar{\alpha}_{s}$ в ИК-области. Отличительной чертой аналитического заряда является его конечность в ИК-области. Это свойство, иногда называемое "замораживанием" (freezing) константы связи, нередко используется для феноменологических целей (см., например, обсуждение в [22]). Экспериментальные свидетельства в пользу регулярного ИК-поведения заряда КХД ухитряются извлекать из физики струй с помошью интегральной характеристики

$$
A(Q)=\frac{1}{Q} \int_{0}^{Q} d k \bar{\alpha}_{s}\left(k^{2}\right)
$$

Эмпирически было установлено [23], что $A(2$ ГэВ $)=0.52 \pm 0.10$.

Нормируем $\bar{\alpha}_{s}$ на массе $\tau$-лептона, полагая $\bar{\alpha}_{s}\left(M_{\tau}^{2}\right)=0.34$. Расчеты величины $A(2$ ГэВ) приведены в табл. 2. Как видно, в рамках АТВ удается единым образом, без введения дополнительных параметров, согласованно описать почти пертурбативную область порядка массы $\tau$-лептона и непертурбативную характеристику (3.1).

3.2. Процесс $e^{+} e^{-}$-аннигиляции в адроны. Применим аналитический подход для анализа процесса $e^{+} e^{-}$-аннигиляции в адроны. Для сравнения с экспериментальными данными будем использовать так называемый метод "размазьвания (smearing)" резонансов, предложенный в работе [24]. В [22] анализ процесса $e^{+} e^{-}$-аннигиляции в адроны был выполнен на основе некоторой “оптимальной” ренормализационной схемы, построенной на основе принципа минимальной чувствительности (PMS) ${ }^{9)}$ [26] и используюшей для оптимизации третий порядок теории возмушений. Наше рассмотрение не основано на какой-либо оптимизации схемного произвола. Более того, как будет показано, схемная зависимость в АТВ оказывается существенно меньшей, чем при использовании обычного подхода, и ее предсказания практически не имеют схемного произвола для всего энергетического интервала.

\footnotetext{
${ }^{9)} \mathrm{PMS}$ - аббревиатура для "principle of minimal sensitivity".
} 
Процедура аналитизации может быть применена и к наблюдаемым величинам, для которых известны подходящие аналитические свойства. Объектом применения АТВ, имеющим множество приложений, является $D$-функция Адлера

$$
D\left(Q^{2}\right)=-Q^{2} \frac{d \Pi\left(-Q^{2}\right)}{d Q^{2}}=3 \sum_{f} Q_{f}^{2}\left[1+d\left(Q^{2}\right)\right]
$$

где П $(s)$ - корреляционная функция, а $d\left(Q^{2}\right)$ - КХД-поправка, имеюшая следующее разложение в ряд РГ-теории возмущений:

$$
d\left(Q^{2}\right)=a\left(Q^{2}\right)\left[1+d_{1} a\left(Q^{2}\right)+d_{2} a^{2}\left(Q^{2}\right)+\cdots\right]
$$

где ${ }^{10)} a=\alpha_{s} / \pi$.

Связь $D$-функции с функцией $R(s)$, определенной как отношение адронного и лептонного сечений для процесса $e^{+} e^{-}$-аннигиляции, дается формулой

$$
D\left(Q^{2}\right)=Q^{2} \int_{0}^{\infty} \frac{d s}{\left(s+Q^{2}\right)^{2}} R(s)
$$

из которой также вытекают свойства $D\left(Q^{2}\right)$ как аналитической функции в $Q^{2}$-плоскости с разрезом вдоль отрицательной полуоси. Определим спектральную плотность $\rho^{\text {эфф }}(\sigma)$ через скачок функции (3.3) на этом разрезе:

$$
\rho^{э ф ф}(\sigma)=\rho^{(1)}(\sigma)+d_{1} \rho^{(2)}(\sigma)+d_{2} \rho^{(3)}(\sigma)+\cdots
$$

Выражение $\rho^{(1)}(\sigma)$ представляет собой спектральную функцию инвариантного заряда, а $\rho^{(k)}(\sigma)$ в выражении (3.5) соответствуют $k$-й степени эффективной связи. Таким образом, аналитическое выражение для КХД-поправки к $D$-функции записывается в виде

$$
d_{\mathrm{ATB}}\left(Q^{2}\right)=\delta_{\mathrm{ATB}}^{(1)}\left(Q^{2}\right)+d_{1} \delta_{\mathrm{ATB}}^{(2)}\left(Q^{2}\right)+d_{2} \delta_{\mathrm{ATB}}^{(3)}\left(Q^{2}\right)+\cdots,
$$

где первое слагаемое $\delta_{\mathrm{ATB}}^{(1)}\left(Q^{2}\right)$ совпадает с аналитическим инвариантным зарядом. Последуюшие слагаемые не сводятся к степеням аналитической константы связи, и, следовательно, метод АТВ приводит к нестепенным разложениям. Анализ свойств таких разложений был дан в работе [12].

Определим КХД-поправку $r(s)$ к функции $R(s)$ так же, как и для $D$-функции в $(3.2)$, и будем использовать соотношения

$$
d\left(Q^{2}\right)=Q^{2} \int_{0}^{\infty} \frac{d s}{\left(s+q^{2}\right)^{2}} r(s), \quad r(s)=-\frac{1}{2 \pi i} \int_{s-i \epsilon}^{s+i \epsilon} \frac{d z}{z} d(-z)
$$

где контур интегрирования в последнем выражении располагается в области аналитичности подынтегрального выражения, обходя разрез вдоль вешественной полуоси.

10) Здесь мы посчитали возможным несколько изменить нормировку константы связи с целью удобства сравнения с работами по соответствующей тематике, в которых, как правило, в качестве инвариантного заряда используется величина $a=\alpha_{s} / \pi$. 
Учет кварковых порогов будем производить с помощью приближенной формулы, предложенной в [24],

$$
R(s)=3 \sum_{f} Q_{f}^{2} \theta\left(s-4 m_{f}^{2}\right) T\left(v_{f}\right)\left[1+g\left(v_{f}\right) r_{f}(s)\right],
$$

где для $v_{f}$ и функций $T(v)$ и $g(v)$ используются выражения

$$
v_{f}=\sqrt{1-\frac{4 m_{f}^{2}}{s}}, \quad T(v)=\frac{v\left(3-v^{2}\right)}{2}, \quad g(v)=\frac{4 \pi}{3}\left[\frac{\pi}{2 v}-\frac{3+v}{4}\left(\frac{\pi}{2}-\frac{3}{4 \pi}\right)\right] .
$$

В АТВ поправка $r_{f}(s)$ выражается через эффективную спектральную плотность

$$
r_{f}(s)=\frac{1}{\pi} \int_{s}^{\infty} \frac{d \sigma}{\sigma} \rho_{f}^{\ni ф \Phi}(\sigma),
$$

где $\rho_{f}^{\text {эфф }}(\sigma)$ определяется через скачок функции $d_{f}\left(Q^{2}\right)$ на физическом разрезе. Соответствующий трехпетлевой вклад записывается в виде

$$
d_{f}\left(Q^{2}\right)=a_{f}\left(Q^{2}\right)\left[1+d_{f}^{(1)} a_{f}\left(Q^{2}\right)+d_{f}^{(2)} a_{f}^{2}\left(Q^{2}\right)\right],
$$

где коэффициенты в $\overline{\mathrm{MS}}$-схеме равны [26]

$$
\begin{gathered}
d_{f}^{(1)}=1.986-0.115 f, \quad d_{f}^{(2)}=18.244-4.216 f+0.086 f^{2}+d_{f}^{\text {singlet }}, \\
d_{f}^{\text {singlet }}=-\frac{1.2395}{3} \frac{\left(\sum_{f^{\prime}}^{f} Q_{f^{\prime}}\right)^{2}}{\sum_{f^{\prime}}^{f} Q_{f^{\prime}}^{2}} .
\end{gathered}
$$

Использование пертурбативных выражений для непосредственного описания экспериментально наблюдаемой величины $R(s)$ при малых $s$ не представляется возможным ввиду наличия пороговых сингулярностей вида $\left(\alpha_{s} / v\right)^{n}$. Воспользуемся предложенным в работе [24] способом "размазки", который позволяет тем не менее провести сравнение с опытом. Идея такого подхода состоит в том, чтобы вместо исходной величины $R(s)$, определенной через корреляционную функцию П следуюшим образом:

$$
R(s)=\frac{1}{2 i}[\Pi(s+i \epsilon)-\Pi(s-i \epsilon)],
$$

ввести величину

$$
R_{\Delta}(s)=\frac{1}{2 i}[\Pi(s+i \Delta)-\Pi(s-i \Delta)]
$$

с некоторым конечным значением $\Delta$. Для значений $s$ вблизи порога величина (3.12) оказывается очень чувствительной к пороговым сингулярностям, при подходе к которым пертурбативное разложение перестает работать. Отступив в комплексной плоскости $q^{2}$ от вешественной оси на конечную величину $\Delta$, как в выражении (3.13), можно рассчитывать на то, что при использовании корректной пертурбативной аппроксимации возможно описать величину (3.13). 


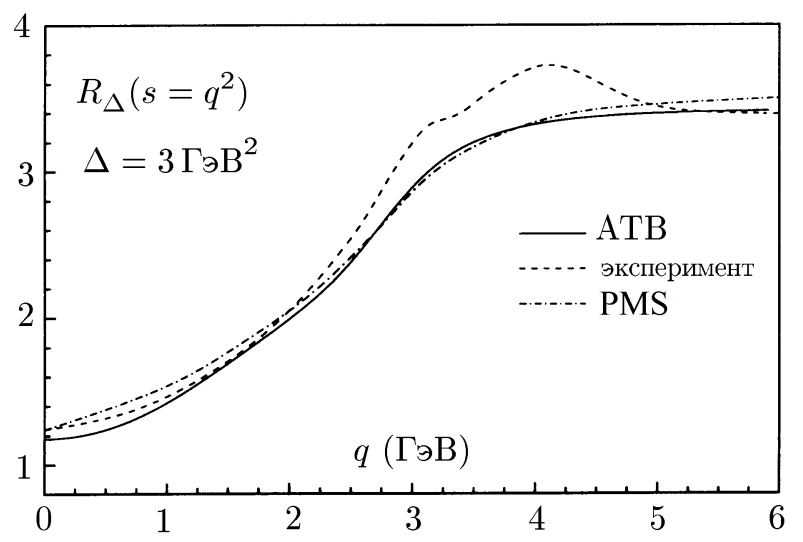

Рис. 6. Величина $R_{\Delta}\left(q^{2}\right)$, соответствующая значению параметра $\Delta=3$ ГэВ $^{2}$. Изображены экспериментальная кривая, результат PMS-оптимизации третьего порядка пертурбативного разложения, полученный в [22], и результат аналитического подхода в третьем порядке.

“Экспериментальная” кривая, соответствующая (3.13), может быть найдена, если, воспользовавшись дисперсионным соотношением для коррелятора П $\left(q^{2}\right)$, записать выражение (3.13) в виде

$$
R_{\Delta}(s)=\frac{\Delta}{\pi} \int_{0}^{\infty} d s^{\prime} \frac{R\left(s^{\prime}\right)}{\left(s-s^{\prime}\right)^{2}+\Delta^{2}} .
$$

Соответствуюшие “экспериментальные” кривые были найдены в работе [22] для некоторых значений параметра $\Delta$, оценки на который сделаны в [24]. Мы будем использовать эти кривые для сравнения с нашими результатами.

Отметим, что прямое использование теории возмущений для описания величины $R_{\Delta}(s)$ опять-таки невозможно. Действительно, $R$-отношение в выражении (3.14), параметризованное с помощью инвариантного заряда с нефизическими сингулярностями, приводит к расходимости интеграла в (3.14). Таким образом, несмотря на то что использование "размазанной” величины (3.14) позволяет обойти затруднение с пороговыми сингулярностями, возникает проблема, связанная с поведением бегушего заряда в ИК-области. Применение АТВ позволяет избежать эту трудность.

На рис. 6 показаны соответствуюшая значению $\Delta=3$ Гэ $\mathrm{B}^{2}$ экспериментальная кривая и кривая, найденная в [24] на основе PMS-оптимизации третьего порядка пертурбативного разложения. На том же рисунке приведен результат нашего расчета в третьем порядке ${ }^{11)}$. Для масштабного параметра в аналитическом подходе взято значение $\Lambda_{\text {ан }}=870 \mathrm{MэB}(f=3)$, полученное из анализа полулептонного $\tau$-распада в рамках ATВ. Для масс кварков приняты следующие, близкие к конституентным (ср. с [27]), значения: $m_{u}=m_{d}=250 \mathrm{MэB}, m_{s}=400 \mathrm{MэB}, m_{c}=1.35$ ГэВ,$m_{b}=4.75$ ГэВ и $m_{t}=174$ ГэВ.

Недавно были получены новые "экспериментальные" данные для $D$-функции [28]. На рис. 7 мы приводим соответствуюшую кривую, а также результаты нашего расчета.

11) Как показано в работе [8], расчет $R_{\Delta}$ в аналитическом подходе приводит к хорошему согласию с экспериментальной кривой уже в первом порядке. 


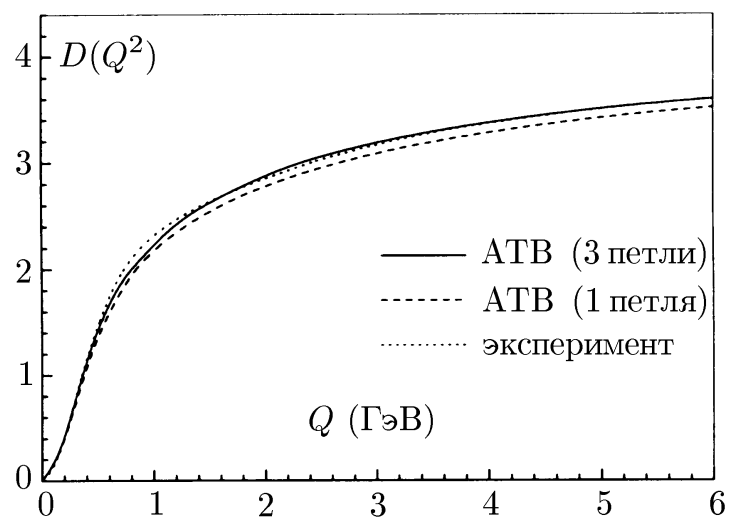

Рис. 7. D-функция.

Рис. 7 демонстрирует, что уже в первом порядке АТВ удается получить хорошее согласие с экспериментальными данными. Это же утверждение, как было отмечено, справедливо и для величины $R_{\Delta}$ на всем энергетическом интервале [8]. Отметим в связи с этим, что при использовании обычного подхода с PMS-оптимизацией петлевой стабильности не наблюдается. Более того, здесь вся "игра" построена на высших приближениях. Так, ситуация с отсутствием или наличием инфракрасно стабильной точки, которая может возникнуть при схемной оптимизации пертурбативного разложения, существенно зависит от рассматриваемой величины (т.е. определяется величиной коэффициентов пертурбативного разложения) [29].

3.3. Зависимость от схемы перенормировки. Неизбежный обрыв ряда ТВ, т.е. аппроксимация физической величины некоторой частичной его суммой, приводит к известной проблеме зависимости результата от ренормализационного предписания. Таким образом, частичная сумма ряда ТВ, используемая для аппроксимации физической величины, обладает зависимостью от выбора схемы перенормировки, которая является источником теоретической неопределенности в описании экспериментальных данных. В КХД такая неопределенность тем больше, чем меньше значения характерных для процесса энергетических параметров. Для решения вопроса об устойчивости получаемых результатов совершенно недостаточно исследовать лишь петлевую стабильность, находясь в рамках какой-либо одной из схем перенормировок, следует рассмотреть также их схемную устойчивость.

Обсудим схемный произвол, возникаюший в $\mathrm{ATB}$, на примере $R$-отношения для процесса $e^{+} e^{-}$-аннигиляции в адроны. Мы рассмотрим класс MS-подобных схем и сравним наши результаты с результатами, полученными в рамках пертурбативного рассмотрения (см., например, [30]).

При переходе от одной схемы перенормировок к другой константа связи преобразуется следуюшим образом:

$$
a^{\prime}=a\left(1+v_{1} a+v_{2} a^{2}+\cdots\right)
$$

Здесь мы ограничимся достигнутым на настояший момент для $D$-функции трехпетле-

6 Теоретическая и математическая физика, т. 120, № 3, 1999 г. 
вым уровнем, когда для КХД-поправки используется приближение

$$
d=a\left(1+d_{1} a+d_{2} a^{2}\right)
$$

в котором бегуший заряд находится как решение ренормгруппового уравнения с трехпетлевой $\beta$-функцией

$$
\beta(a)=\mu^{2} \frac{\partial a}{\partial \mu^{2}}=-b a^{2}\left(1+b_{1} a+b_{2} a^{2}\right),
$$

где

$$
b=\frac{33-2 f}{6}, \quad b_{1}=\frac{153-19 f}{66-4 f}, \quad b_{2}^{\overline{\mathrm{MS}}}=\frac{77139-15099 f+325 f^{2}}{288(33-2 f)} .
$$

Трехпетлевой коэффициент $\beta$-функции $b_{2}$, а также коэффициенты разложения $d_{1}$ и $d_{2}$ в (3.16) зависят от выбора схемы перенормировки. При схемном преобразовании (3.15) они изменяются следуюшим образом:

$$
\begin{aligned}
& b_{2}^{\prime}=b_{2}-v_{1}^{2}-b_{1} v_{1}+v_{2}, \\
& d_{1}^{\prime}=d_{1}-v_{1}, \\
& d_{2}^{\prime}=d_{2}-2\left(d_{1}-v_{1}\right) v_{1}-v_{2} .
\end{aligned}
$$

Итак, в представлении (3.16) каждый член испытывает преобразование, и в результате приходим к новой функции

$$
d^{\prime}=a^{\prime}\left(1+d_{1}^{\prime} a^{\prime}+d_{2}^{\prime} a^{\prime 2}\right),
$$

где константа $a^{\prime}$ вычисляется с новой $\beta$-функцией, в которой трехпетлевой коэффициент $b_{2}$ заменяется штрихованным $b_{2}^{\prime}$.

Учитывая закон преобразования масштабного параметра $\Lambda^{\prime}=\Lambda \exp \left(v_{1} / b\right)[31]$ и формулы (3.19), находим два схемных инварианта [25]

$$
\rho_{1}=\frac{b}{2} \ln \frac{Q^{2}}{\Lambda^{2}}-d_{1}, \quad \rho_{2}=b_{2}+d_{2}-b_{1} d_{1}-d_{1}^{2} .
$$

Нормируем импульсную шкалу на значение $\Lambda_{\overline{\mathrm{MS}}}$. В этом случае инвариантный заряд в произвольной схеме находится из уравнения

$$
\frac{b}{2} \ln \left(\frac{Q^{2}}{\Lambda \frac{2}{\mathrm{MS}}}\right)=d_{1}^{\overline{\mathrm{MS}}}-d_{1}+\Phi\left(a, b_{2}\right),
$$

где

$$
\Phi\left(a, b_{2}\right)=\frac{1}{a}-b_{1} \ln \frac{1+b_{1} a}{\beta_{0} a}+b_{2} \int_{0}^{a} \frac{d x}{\left(1+b_{1} x\right)\left(1+b_{1} x+b_{2} x^{2}\right)} .
$$

Хотя каких-либо аргументов общего характера, которые позволили бы с самого начала отдать предпочтение той или иной схеме перенормировок, не существует, тем не менее можно определить класс "естественных" схем, которые выглядят разумными на 


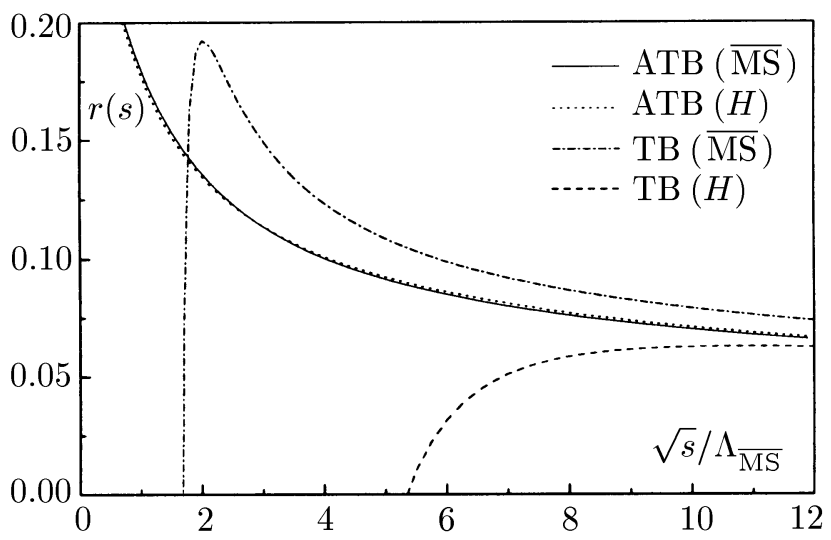

Рис. 8. Графики функции $r(s)$, вычисленной в теории возмущений (ТВ) и в аналитическом подходе (АТВ) для двух схем перенормировки $H$ и $\overline{\mathrm{MS}}$, имеющих примерно один и тот же индекс сокрашений $C_{R} \simeq 2$.

рассматриваемом трехпетлевом уровне. Критерий для этого был предложен в работе [32]. Следует ограничиться такими схемами, для которых сокрашения между слагаемыми во втором схемном инварианте (3.21) не слишком велики. Количественно такой критерий можно соотнести с величиной индекса сокрашений

$$
C=\frac{1}{\left|\rho_{2}\right|}\left(\left|b_{2}\right|+\left|d_{2}\right|+d_{1}^{2}+\left|d_{1}\right| b_{1}\right) .
$$

Следует, конечно, понимать определенную условность такого рассмотрения, в особенности когда речь пойдет о минимальной величине индекса сокрашений.

Задавшись некоторым максимальным значением индекса сокрашений $C_{\max }$, можно изучить стабильность получаемых результатов, перебирая схемы с индексом $C \leqslant$ $C_{\text {max }}$. Примем в качестве $C_{\max }$ индекс, который соответствует оптимальной PMS-схеме. В этом случае мы имеем сравнительно узкий класс "разрешенных" схем, граница которого определяется максимальным индексом $C_{\mathrm{PMS}}$.

Для $R(s)$ индекс сокрашений $C_{R}$ вычисляется по известным коэффициентам $r_{1}$ и $r_{2}$ пертурбативного разложения поправки $r=a\left(1+r_{1} a+r_{2} a^{2}\right)$. Для PMS-схемы он оказывается равным $C_{\mathrm{PMS}} \simeq 2$. Чтобы продемонстрировать возникаюший схемный произвол, выберем из этого класса две схемы. В качестве первой возьмем схему $H$ с параметрами $r_{1}^{(H)}=-3.2$ и $b_{2}^{(H)}=0$ (схема т' Хоофта). В качестве второй - $\overline{\mathrm{MS}}$-схему, которой соответствуют параметры $r_{1}^{(\overline{\mathrm{MS}})}=1.64$ и $b_{2}^{(\overline{\mathrm{MS}})}=4.47$. Обе эти схемы имеют близкий друг к другу и к граничному индекс сокращений $C_{H} \simeq C_{\overline{\mathrm{MS}}} \simeq C_{\mathrm{PMS}} \simeq 2$.

На рис. 8 изображена КХД-поправка $r(s)$ как функция $\sqrt{s} / \Lambda_{\overline{\mathrm{MS}}}$, вычисленная в теории возмущений и в аналитическом подходе для двух схем перенормировки $H$ и $\overline{\mathrm{MS}}$, характеризуюшихся примерно одним и тем же индексом сокрашений $C_{R} \simeq 2$. Как видно из этого рисунка, применение аналитического подхода позволяет кардинальным образом уменьшить схемный произвол.

Резкое уменьшение схемной зависимости в АТВ имеет место и для других процессов, таких как инклюзивный $\tau$-распад [11] и правила сумм Бьеркена и Гросса-Ллювеллина 


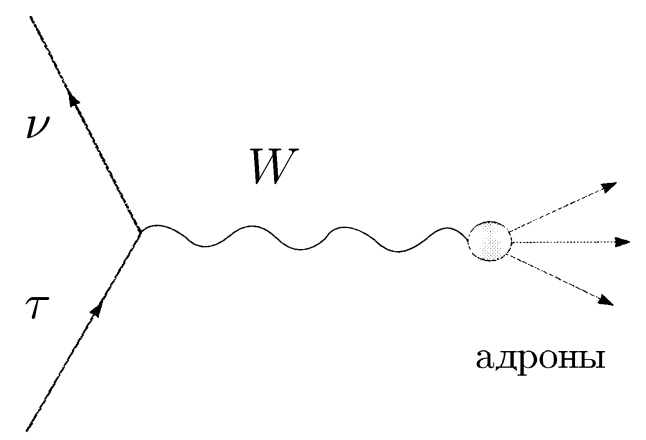

Рис. 9. Диаграмма инклюзивного распада $\tau$-лептона.

Смита для неупругого лептон-адронного рассеяния [13, 14]. Таким образом, в аналитическом подходе достигнутый на сегодняшний день для многих физических процессов трехпетлевой уровень оказывается практически инвариантным по отношению к выбору ренормировочного предписания.

3.4. Инклюзивный распад $\tau$-лептона. Инклюзивный $\tau$-распад (соответствуюшая диаграмма изображена на рис.9) дает возможность низкоэнергетического тестирования КХД. Масса $\tau$-лептона $M_{\tau}=1777_{-0.26}^{+0.29} \mathrm{MэB} \mathrm{[33],} \mathrm{с} \mathrm{одной} \mathrm{стороны,} \mathrm{достаточ-}$ но велика, чтобы были возможны адронные моды распада, а с другой стороны, в шкале хромодинамических масштабов ее значение мало и находится в низкоэнергетической области. Теоретическое описание инклюзивного $\tau$-распада в принципе возможно без каких-либо модельных допущений, что важно для надежного определения низкоэнергетического значения $\bar{\alpha}_{s}\left(M_{\tau}^{2}\right)$ из экспериментальных данных. Основной величиной для изучения является $R_{\tau}$-отношение

$$
R_{\tau}=\frac{\Gamma\left[\tau^{-} \rightarrow \nu_{\tau}+\operatorname{aдроны}(\gamma)\right]}{\Gamma\left[\tau^{-} \rightarrow \nu_{\tau} e^{-} \bar{\nu}_{e}(\gamma)\right]}
$$

которое в современных экспериментах измеряется с процентной точностью.

Исходным для теоретического анализа служит выражение

$$
R_{\tau}=2 \int_{0}^{M_{\tau}^{2}} \frac{d s}{M_{\tau}^{2}}\left(1-\frac{s}{M_{\tau}^{2}}\right)^{2}\left(1+\frac{2 s}{M_{\tau}^{2}}\right) \widetilde{R}(s),
$$

где $\widetilde{R}(s)$ определяется мнимой частью адронного коррелятора

$$
\Pi(s)=\sum_{q=d, s}\left|V_{u q}\right|^{2}\left[\Pi_{u q, V}(s)+\Pi_{u q, A}(s)\right] .
$$

Здесь $V_{u q}$ - элементы матрицы Кобаяши-Маскава. В рассматриваемом нами безмассовом случае векторный $\Pi_{u q, V}$ и аксиально-векторный $\Pi_{u q, A}$ адронные корреляторы 


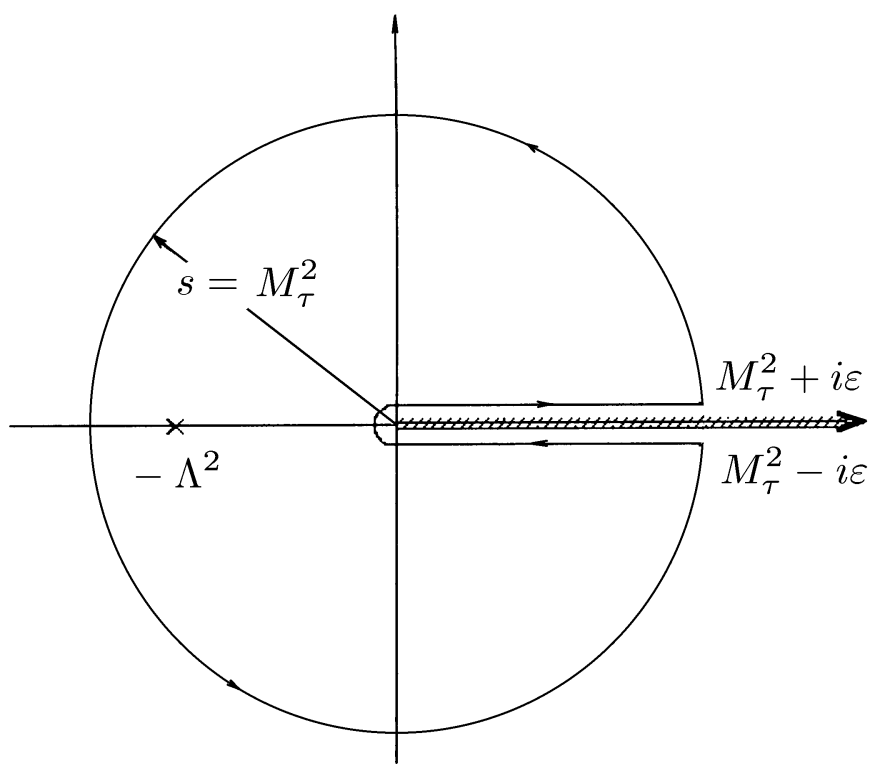

Рис. 10. Переход к контурному представлению для $R_{\tau}$.

совпадают и функция $\widetilde{R}(s)$ равна отношению $R(s)$ для процесса $e^{+} e^{-}$-аннигиляции в адроны.

При обычном анализе $\tau$-распада сразу же возникает трудность применения исходной формулы (3.26), поскольку параметризация функции $R(s)$ пертурбативной $\bar{\alpha}_{s}$ с нефиизическими особенностями приводит к сингулярностям подынтегрального выражения. "Выход" из этого затруднения, предложенный в [34], состоит в следуюшем. Интеграл (3.26) представляют в виде комбинации интегралов по берегам разреза в комплексной плоскости $s$ (см. рис. 10). Затем по теореме Коши этот интеграл "преобразовывают" к интегралу по контуру $|s|=M_{\tau}^{2}$. В итоге после интегрирования по частям возникает контурное представление $R_{\tau}$ через $D$-функцию:

$$
R_{\tau}=\frac{1}{2 \pi i} \oint_{|z|=1} \frac{d z}{z}(1-z)^{3}(1+z) D\left(M_{\tau}^{2} z\right)
$$

Переход от исходного выражения (3.26) к контурному представлению (3.28) опирается на определенные аналитические свойства коррелятора, которые нарушены при обычном анализе. Таким образом, наличие правильных аналитических свойств, которые обеспечивает аналитический подход, оказывается важным для непротиворечивого описания инклюзивного $\tau$-распада.

Рассмотрим описание этого процесса в АТВ [9]. Выделим вклад сильных взаимодействий $\Delta_{\tau}$ в $R_{\tau}$-отношение

$$
R_{\tau}=R_{\tau}^{(0)}\left(1+\Delta_{\tau}\right)
$$

где $R_{\tau}^{(0)}$ - известньй фактор, включающий электрослабые поправки. 
Выражение $\Delta_{\tau}$ через эффективную спектральную функцию записывается в виде

$$
\Delta_{\tau}=\frac{d_{1}}{\pi} \int_{0}^{\infty} \frac{d \sigma}{\sigma} \rho^{\text {эфф }}(\sigma)-\frac{d_{1}}{\pi} \int_{0}^{M_{\tau}^{2}} \frac{d \sigma}{\sigma}\left(1-\frac{\sigma}{M_{\tau}^{2}}\right)^{3}\left(1+\frac{\sigma}{M_{\tau}^{2}}\right) \rho^{\text {эфф }}(\sigma) .
$$

Благодаря свойству универсальности интеграл в первом слагаемом может быть выражен через $a_{\text {ан }}(0)$. Спектральная функция в двухпетлевом приближении имеет вид

$$
\rho^{э ф ф}(\sigma)=\varrho(\sigma)+\frac{1}{\beta_{0}^{2}} \frac{d_{2}}{d_{1}} \frac{2 R(L) I(L)}{\left[R^{2}(L)+I^{2}(L)\right]^{2}},
$$

где спектральная плотность инвариантного заряда $\varrho(\sigma)$ определена в $(2.8)$, а функции $R(L)$ и $I(L)$ заданы выражениями (2.9). Подстановка (3.31) в (3.30) позволит вычислить вклад сильных взаимодействий $\Delta_{\tau}$ в терминах масштабного параметра $\Lambda$.

Используя экспериментальное значение $R_{\tau}=3.633 \pm 0.031$ [33], получаем $\alpha\left(M_{\tau}^{2}\right)=$ $0.400 \pm 0.026$ и соответствуюшее ему значение масштабного параметра $\Lambda_{\text {ан }}^{(3)}=935 \pm$ 159 МэВ. Эти значения оказываются бо́льшими, чем получаемые при обработке по ТВ с использованием контурного представления [35]. Причина состоит в том, что присущие аналитическому подходу непертурбативные поправки вносят в величину $\Delta_{\tau}$ отрицательный вклад $[9,10]$. Таким образом, для того чтобы получить одно и то же значение $\Delta_{\tau}$ в ТВ и в аналитическом подходе, в последнем необходимо увеличить вклад “пертурбативной составляющей” за счет увеличения величины $\Lambda$. Анализ инклюзивного $\tau$-распада на трехпетлевом уровне в АТВ был выполнен в работе [36]. При этом извлекаемое значение параметра $\Lambda$ оказалось несколько меньшим: $\Lambda_{\mathrm{aн}}^{(3)}=871 \pm 155$ МэВ. В этой же работе показана схемная устойчивость такого рассмотрения. Следует отметить, что величина $\Lambda_{\text {ан }}$ оказывается очень чувствительной к экспериментальному значению $R_{\tau}$. Так, если использовать $R_{\tau}=3.559 \pm 0.035$ [37], то получим $\Lambda_{\text {ан }}^{(3)}=640 \pm 127$ МэВ, что отвечает заметно меньшему значению инвариантного заряда на массе $M_{\tau}$ (см. табл. 1$)$.

\section{4. АНАЛИТИЧЕСКИЙ ПОДХОД ДЛЯ НЕУПРУГОГО ЛЕПТОН-А ДРОННОГО РАССЕЯНИЯ}

В этом разделе мы изложим теоретическую основу возможного применения нашего аналитического описания характеристик процесса неупругого лептон-адронного рассеяния. Ключевой момент нашего построения - использование аналитических по $Q^{2}$ свойств моментов структурных функций - потребует некоторой модификации общепринятого формализма и, в частности, замены обычных, бьеркеновских, моментов $M_{n}\left(Q^{2}\right)$ на некоторые модифицированные моменты $\mathcal{M}_{n}\left(Q^{2}\right)$ по новой скейлинговой переменной, учитьвающей кинематические массовые зависимости. Мы будем исходить из интегрального представления Йоста-Лемана (см., например, $§ 55$ книги [5]) для фурье-образа соответствуюшего матричного элемента.

4.1. Представление Йоста-Лемана. Структурные функции процесса неупругого лептон-адронного рассеяния зависят от двух аргументов, и соответствуюшие представления, которые аккумулируют фундаментальные свойства теории, такие как релятивистская инвариантность, спектральность и причинность, будут иметь по сравнению с представлениями для функций одного аргумента более сложный вид. В литературе известны два таких представления. Мы будем использовать 4-мерное интегральное 


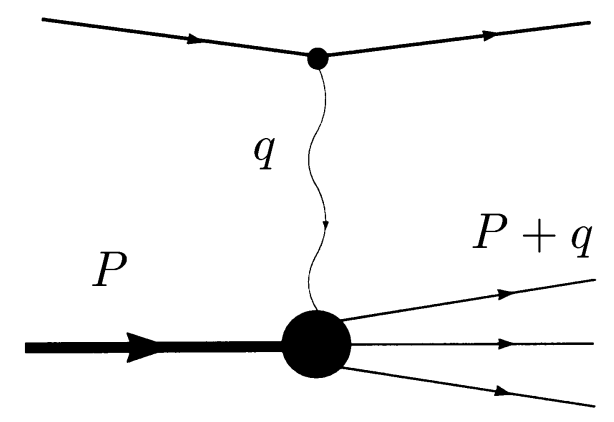

Рис. 11. Диаграмма глубоконеупругого лептон-адронного рассеяния в приближении однофотонного обмена.

представление, предложенное Йостом и Леманом [38] для так называемого симметричного случая ${ }^{12)}$. Применению этого представления для изучения автомодельной асимптотики структурных функций были посвяшены работы Боголюбова, Владимирова и Тавхелидзе [4], некоторыми результатами и обозначениями которых мы будем пользоваться. Доказательство представления Йоста-Лемана базируется на самых общих свойствах теории, таких как ковариантность, эрмитовость, спектральность и причинность (см. книгу [5]; некоторые математические вопросы в связи с представлением Йоста-Лемана-Дайсона рассмотрены также в книгах [40, 41]).

Для определенности будем говорить о неупругом рассеянии заряженных лептонов (электронов, мюонов) на нуклонах, т.е. рассматривать процесс $\ell+N \rightarrow \ell+$ адроны. В низшем порядке по электромагнитной константе связи (однофотонный обмен) этот процесс изображен на рис. 11, из которого также ясны используемые обозначения. В неполяризованном случае сечение процесса определяется адронным тензором

$$
W_{\mu \nu}(q, P)=\frac{1}{4 \pi} \sum_{\sigma} \int d x \exp (i q \cdot x)\left\langle P, \sigma\left|\left[J_{\mu}\left(\frac{x}{2}\right), J_{\nu}\left(-\frac{x}{2}\right)\right]\right| P, \sigma\right\rangle,
$$

образованным коммутатором токов, причем сумма берется по поляризациям нуклона.

Релятивистская инвариантность и сохранение электромагнитного тока приводят к параметризации тензора (4.1) двумя структурными функциями $w_{1}$ и $w_{2}$ :

$$
\begin{aligned}
W_{\mu \nu}(q, P)= & \left(-g_{\mu \nu}+\frac{q_{\mu} q_{\nu}}{q^{2}}\right) w_{1}(q, P)+ \\
& +\frac{1}{M^{2}}\left(p_{\mu}-\frac{P \cdot q}{q^{2}} q_{\mu}\right)\left(p_{\nu}-\frac{P \cdot q}{q^{2}} q_{\nu}\right) w_{2}(q, P),
\end{aligned}
$$

здесь $M=\sqrt{P^{2}}$ - масса нуклона.

Перечис лим основные свойства функций $w$, вытекаюшие из обших принципов локальной КТП:

\footnotetext{
12) Более общий случай был рассмотрен Дайсоном [39]. Вследствие этого подобные представления часто именуют представлениями Йоста-Лемана-Дайсона.
} 
- свойство ковариантности означает, что функции $w$ зависят от двух скалярных аргументов, в качестве которых выбираются $\nu=P \cdot q$ и $Q^{2}=-q^{2}$ :

$$
w(q, P)=W\left(\nu, Q^{2}\right)
$$

- свойство спектральности записывается следуюшим образом:

$$
W\left(\nu, Q^{2}\right)=0 \quad \text { при } \quad \frac{Q^{2}}{2 \nu}=x>1,
$$

где использована безразмерная бьеркеновская переменная, которая в физической области процесса при $(q+P)^{2}>M^{2}$ кинематически ограничена интервалом $0<x<1$;

- структурная функция параметризует сечение рассеяния и является вешественной (свойство вещественности):

$$
W\left(\nu, Q^{2}\right)=W^{*}\left(\nu, Q^{2}\right)
$$

- эрмитовость оператора тока приводит к свойству (анти) симметрии

$$
W\left(-\nu, Q^{2}\right)=-W\left(\nu, Q^{2}\right)
$$

- исчезновение коммутатора токов для пространственноподобных интервалов вследствие локальной коммутативности токов дает условие причинности

$$
\int \frac{d q}{(2 \pi)^{4}} \exp (-i q z) W(q, P)=0 \quad \text { для } \quad z^{2}<0 .
$$

Для функции $W\left(\nu, Q^{2}\right)$, удовлетворяюшей всем этим условиям, существует вещественная обобщенная функция медленного роста $\psi\left(\mathbf{u}, \lambda^{2}\right)$ такая, что справедливо интегральное представление Йоста-Лемана, которое в системе покоя нуклона принимает вид [4]

$$
W\left(\nu, Q^{2}\right)=\varepsilon\left(q_{0}\right) \int d \mathbf{u} d \lambda^{2} \delta\left[q_{0}^{2}-(M \mathbf{u}-\mathbf{q})^{2}-\lambda^{2}\right] \psi\left(\mathbf{u}, \lambda^{2}\right),
$$

причем носитель функции $\psi\left(\mathbf{u}, \lambda^{2}\right)$ сосредоточен на множестве

$$
\rho=|\mathbf{u}| \leqslant 1, \quad \lambda^{2} \geqslant M^{2}\left(1-\sqrt{1-\rho^{2}}\right)^{2} .
$$

Для рассматриваемого процесса физические значения переменных $\nu$ и $Q^{2}$ положительны. Поэтому можно отвлечься от фактора $\varepsilon\left(q_{0}\right)=\varepsilon(\nu)$, оставив то же обозначение $W\left(\nu, Q^{2}\right)$. Принимая во внимание вытекающую из ковариантности радиальную симметрию весовой функции $\psi\left(\mathbf{u}, \lambda^{2}\right)=\psi\left(\rho, \lambda^{2}\right)[4]$, запишем для $W$ представление Йоста-Лемана в ковариантной форме:

$$
\begin{aligned}
W\left(\nu, Q^{2}\right)= & \int_{0}^{1} d \rho \rho^{2} \int_{\lambda_{\min }^{2}}^{\infty} d \lambda^{2} \int_{-1}^{1} d z \times \\
& \times \delta\left(Q^{2}+M^{2} \rho^{2}+\lambda^{2}-2 z \rho \sqrt{\nu^{2}+M^{2} Q^{2}}\right) \psi\left(\rho, \lambda^{2}\right),
\end{aligned}
$$


где

$$
\lambda_{\min }^{2}=M^{2}\left(1-\sqrt{1-\rho^{2}}\right)^{2} .
$$

4.2. Аналитические моменты структурных функций. Как это следует из представления (4.4), естественной скейлинговой переменной оказывается переменная

$$
s=\frac{1}{2} \sqrt{\frac{Q^{2}\left(Q^{2}+4 M^{2}\right)}{\nu^{2}+M^{2} Q^{2}}}=x \sqrt{\frac{Q^{2}+4 M^{2}}{Q^{2}+4 M^{2} x^{2}}},
$$

аккумулируюшая корневую структуру из аргумента $\delta$-функции. В то же время в физической области процесса переменная $s$ изменяется так же, как и бьеркеновская переменная $x$, т.е. в пределах от нуля до единицы. Переменная $s$ содержит зависимость от массы мишени (нуклона) и отличается не только от бьеркеновской, но и от нахтмановской переменной [42]

$$
\xi=\frac{2 x}{1+\sqrt{1+x^{2} 4 M^{2} / Q^{2}}},
$$

которая иногда применяется при кинематическом учете массовых эффектов процесса неупругого рассеяния. Однако лишь переменная $s$ приводит к моментам, которые обладают подходящими для нас аналитическими свойствами по $Q^{2}$.

Для того чтобы установить эти свойства, выполним в (4.4) интегрирование по $z$,

$$
\begin{aligned}
W\left(\nu, Q^{2}\right)= & \frac{s}{Q^{2} \sqrt{1+4 M^{2} / Q^{2}}} \int_{0}^{1} d \rho \rho \times \\
& \times \int_{\lambda_{\min }^{2}}^{\infty} d \lambda^{2} \theta\left[Q^{2} \rho^{2}-s \frac{\left(Q^{2}+M^{2} \rho^{2}+\lambda^{2}\right)}{\sqrt{1+4 M^{2} / Q^{2}}}\right] \psi\left(\rho, \lambda^{2}\right) .
\end{aligned}
$$

Определим теперь модифицированные $s$-моменты структурных функций (ср. с [43])

$$
\mathcal{M}_{n}\left(Q^{2}\right)=\frac{1}{\left(1+4 M^{2} / Q^{2}\right)^{(n-1) / 2}} \int_{0}^{1} d s s^{n-2} W\left(\nu, Q^{2}\right) .
$$

Подставляя сюда $W\left(\nu, Q^{2}\right)$ в виде $(4.7)$, получаем

$$
\mathcal{M}_{n}\left(Q^{2}\right)=\frac{\left(Q^{2}\right)^{n-1}}{n} \int_{0}^{1} d \rho \rho^{n+1} \int_{0}^{\infty} d \sigma \frac{\theta\left(\sigma-\sigma_{\min }\right)}{\left(Q^{2}+\sigma\right)^{n}} \psi\left(\rho, \sigma-M^{2} \rho^{2}\right),
$$

где $\sigma=\lambda^{2}+M^{2} \rho^{2}$ и $\sigma_{\min }=2 M^{2}\left(1-\sqrt{1-\rho^{2}}\right)$.

Вводя весовую функцию

$$
m_{n}(\sigma)=\frac{1}{n} \int_{0}^{1} d \rho \rho^{n+1} \theta\left(\sigma-\sigma_{\min }\right) \psi\left(\rho, \sigma-M^{2} \rho^{2}\right),
$$

приходим к представлению для $s$-моментов

$$
\mathcal{M}_{n}\left(Q^{2}\right)=\left(Q^{2}\right)^{n-1} \int_{0}^{\infty} d \sigma \frac{m_{n}(\sigma)}{\left(\sigma+Q^{2}\right)^{n}}
$$


из которого вытекает аналитичность $\mathcal{M}_{n}\left(Q^{2}\right)$ в комплексной плоскости $Q^{2}$, разрезанной вдоль отрицательной полуоси, т.е. аналитичность типа Челлена-Лемана.

Заметим здесь, что в работе [44] на основе интегрального представления ДезераГильберта-Сударшана [45] сделано аналогичное утверждение об аналитичности типа Челлена-Лемана в отношении $x$-моментов. Однако статус упомянутого представления в КТП оказывается менее определенным - его не удается получить исходя лишь из основных принципов теории.

Взаимосвязь между моментами (4.8) и обычными бьеркеновскими моментами

$$
M_{n}\left(Q^{2}\right)=\int_{0}^{1} d x x^{n-2} W\left(\nu, Q^{2}\right)
$$

может быть выражена разложениями

$$
\begin{aligned}
& \mathcal{M}_{n}\left(Q^{2}\right)=\frac{1}{\Gamma[(n+1) / 2]} \sum_{k=0}^{\infty} \frac{\Gamma[k+(n+1) / 2]}{k !}\left(-\frac{4 M^{2}}{Q^{2}}\right)^{k} M_{n+2 k}\left(Q^{2}\right), \\
& M_{n}\left(Q^{2}\right)=\frac{1}{\Gamma[(n+1) / 2]} \sum_{k=0}^{\infty} \frac{\Gamma[k+(n+1) / 2]}{k !}\left(\frac{4 M^{2}}{Q^{2}}\right)^{k} \mathcal{M}_{n+2 k}\left(Q^{2}\right) .
\end{aligned}
$$

В асимптотической области, соответствуюшей большим значениям переданного импульса $Q^{2}$, когда возможно пренебречь степенными поправками вида $1 /\left(Q^{2}\right)^{n}, x-, s$ и $\xi$-моменты совпадают друг с другом. Вне же асимптотической области, когда речь идет об изучении вклада высших твистов, различие между этими определениями моментов следует принимать во внимание.

4.3. Дисперсионное соотношение и операторное разложение. Для установления связи с операторным разложением получим исходя из представления Йоста-Лемана дисперсионное соотношение для амплитуды комптоновского рассеяния вперед по новой переменной (4.6). Отвечающий представлению (4.4) матричный элемент процесса запишем в виде

$$
\begin{aligned}
T\left(\nu, Q^{2}\right)= & \frac{1}{\pi} \int_{0}^{1} d \rho \rho^{2} \int_{\lambda_{\text {min }}^{2}}^{\infty} d \lambda^{2} \times \\
& \times \int_{-1}^{1} \frac{\psi\left(\rho, \lambda^{2}\right) d z}{Q^{2}+M^{2} \rho^{2}+\lambda^{2}-2 z \rho \sqrt{\nu^{2}+M^{2} Q^{2}}-i \epsilon}
\end{aligned}
$$

В комплексной плоскости $\nu^{2}$ функция $T\left(\nu, Q^{2}\right)$ имеет разрез вдоль положительной части вещественной оси, начинающийся с $\nu_{\min }^{2}$, значение которого определяется условием

$$
\sqrt{\nu_{\min }^{2}+M^{2} Q^{2}}=\min _{\{\lambda, \rho, z\}}\left|\frac{Q^{2}+M^{2} \rho^{2}+\lambda^{2}}{2 z \rho}\right| .
$$

С учетом (4.5) и пределов изменения переменной интегрирования $z$ в (4.12) это выражение упрощается:

$$
\sqrt{\nu_{\min }^{2}+M^{2} Q^{2}}=\min _{\{\rho\}} \frac{Q^{2}+2 M^{2}\left(1-\sqrt{1-\rho^{2}}\right)}{2 \rho}
$$


и приводит к $\nu_{\min }^{2}=\left(Q^{2} / 2\right)^{2}$. Таким образом, искомое дисперсионное соотношение имеет вид

$$
T\left(\nu, Q^{2}\right)=\frac{1}{\pi} \int_{Q^{4} / 4}^{\infty} \frac{d \nu_{1}^{2}}{\nu_{1}^{2}-\nu^{2}-i \epsilon} W\left(\nu_{1}, Q^{2}\right) .
$$

Отметим, что в терминах бьеркеновской переменной $x$ соотношение (4.14) представляется в виде

$$
T\left(\nu, Q^{2}\right)=\frac{2}{\pi} \int_{0}^{1} \frac{d x_{1}}{x_{1}} \frac{1}{1-\left(x_{1} / x\right)^{2}} W\left(\nu_{1}, Q^{2}\right) .
$$

Это выражение определяет простые свойства амплитуды $T\left(x, Q^{2}\right)$ в комплексной $x$-плоскости и удобно для связи с операторным разложением.

При рассмотрении следствий представления Йоста-Лемана, как отмечалось вьше, естественной скейлинговой переменной является $s$. В этом случае возникает аналогичная структура дисперсионного интеграла ${ }^{13)}$

$$
T\left(\nu, Q^{2}\right)=\frac{2}{\pi} \int_{0}^{1} \frac{d s_{1}}{s_{1}} \frac{1}{1-\left(s_{1} / s\right)^{2}} W\left(\nu_{1}, Q^{2}\right) ; \quad \nu^{2}=Q^{2}\left[\frac{Q^{2}+4 M^{2}}{4 s^{2}}-M^{2}\right] .
$$

Тождество структур дисперсионных соотношений по переменным $x$ и $s$ позволяет установить связь аналитических моментов (4.8) с разложением произведения операторов токов, которое используется для нахождения $Q^{2}$-эволюции моментов структурных функций. Моменты (4.11) соответствуют тому, что в матричных элементах оператора $\left\langle P\left|\widehat{O}_{\mu_{1} \ldots \mu_{n}}\right| P\right\rangle$ учитываются лишь лоренц-структуры вида $P_{\mu_{1}} \ldots P_{\mu_{n}}$. В этом случае применение операторного разложения для комптоновской амплитуды приводит к разложению по степеням $(q \cdot P) / Q^{2}$, т.е. к разложению по обратным степеням $x$. Такое же разложение по обратным степеням $x$ можно провести и в дисперсионном интеграле (4.15). Коэффициенты при этом будут определяться $x$-моментами. Сравнение двух рядов и дает искомую связь $x$-моментов и операторного разложения.

В общем случае симметричный матричный элемент $\left\langle P\left|\widehat{O}_{\mu_{1} \ldots \mu_{n}}\right| P\right\rangle$ содержит следуюшие лоренц-структуры: $\left\{P_{\mu_{1}} \ldots P_{\mu_{n}}\right\}, M^{2} g_{\mu_{i} \mu_{j}}\left\{P_{\mu_{1}} \ldots P_{\mu_{n-2}}\right\}$ и т.д. Моменты по переменной $\xi$ соответствуют выбору такого операторного базиса, в котором разложение осушествляется по бесследовым тензорам, т.е. таким, что свертка $g_{\mu_{i} \mu_{j}}$ с $\left\langle P\left|\widehat{O}_{\mu_{1} \ldots \mu_{n}}\right| P\right\rangle$ по любым двум индексам обрашается в нуль. При этом, очевидно, лоренш-структура матричных элементов $\left\langle P\left|\widehat{O}_{\mu_{1} \ldots \mu_{n}}\right| P\right\rangle$ фиксируется однозначно.

Дисперсионное представление (4.16) позволяет провести разложение комптоновской амплитуды по обратным степеням переменной $s$. Если выбрать операторный базис таким образом, чтобы произвольная свертка тензора $\left\langle P\left|\widehat{O}_{\mu_{1} \ldots \mu_{n}}\right| P\right\rangle$ с импульсом нуклона $P_{\mu_{i}}$ обрашалась в нуль, то операторное разложение приведет к степенному ряду для амплитуды комптоновского рассеяния вперед с параметром разложения $q_{\mu} q_{\nu}\left(P_{\mu} P_{\nu}-\right.$ $\left.g_{\mu \mu} P^{2}\right) /\left(q^{2}\right)^{2}$, что соответствует разложению в дисперсионном интеграле (4.16) по степеням $1 / s^{2}$. Таким образом устанавливается связь аналитических $s$-моментов с операторным разложением. Подчеркнем, что требование ортогональности симметричного тензора $\left\langle P\left|\widehat{O}_{\mu_{1} \ldots \mu_{n}}\right| P\right\rangle$ импульсу нуклона $P_{\mu_{i}}$ определяет его лоренш-структуру однозначным образом.

13) Отметим, что при использовании иных скейлинговых переменных, например нахтмановской, такая структура может разрушаться. 


\section{5. ЗАКЛЮЧЕНИЕ}

В работе рассмотрена аналитическая формулировка КХД, в которой аналитизированные РГ-решения для инвариантных функций связи, функций Грина и матричных элементов свободны от нефизических особенностей. Важным свойством этой формулировки является обнаруженная стабильность аналитического инвариантного заряда по отношению к высшим петлевым поправкам для всего интервала $Q^{2}$. Ключевым моментом здесь является наличие универсального предельного значения $\bar{\alpha}_{\text {ан }}(0)=4 \pi / \beta_{0}$, инвариантного по отношению к многопетлевым поправкам. Эта константа не зависит от параметра $\Lambda_{\mathrm{KX}}$ д и определяется лиш обшими симметрийными свойствами лагранжиана. Поэтому семейство кривых $\bar{\alpha}_{\text {ан }}\left(Q^{2} / \Lambda^{2}\right)$ для различных значений параметра $\Lambda$ представляет собой пучок с общей точкой $\bar{\alpha}_{\text {ан }}(0)=4 \pi / \beta_{0}$ (эта картина не зависит от числа петель).

Инвариантная аналитическая формулировка сушественно модифицирует поведение $\bar{\alpha}_{\text {ан }}(x)$ в ИК-области, делая ее устойчивой к высшим петлевым поправкам. Двухпетлевое приближение отличается от однопетлевого не более чем на $\simeq 10 \%$ в области малых $Q^{2}$, а трехпетлевое отличается от двухпетлевого лишь на $\simeq 1 \%$. Такая ситуация кардинально отличается от ситуации в обычной ренормгрупповой ТВ, для которой характерна сильная неустойчивость по отношению к последуюшим петлевым поправкам в области малых $Q^{2} \simeq \Lambda^{2}$. Отметим еще, что поддержка корректных аналитических свойств по $Q^{2}$ оказывается сушественной для самосогласованного определения эффективной функции связи во времениподобной области [15]. При описании конкретных процессов, например инклюзивного распада $\tau$-лептона, непротиворечивое рассмотрение возможно [9] только лишш при наличии отмеченных выше аналитических свойств.

Для описания физических величин в рамках нового подхода имеются по крайней мере две возможности. Наиболее простая из них состоит в замене $\bar{\alpha}_{s}(x) \rightarrow \bar{\alpha}_{\text {ан }}(x)$ в явных, "обработанных" методом РГ выражениях для наблюдаемых, точнее, в связанных с ними величинах, определенных в пространственноподобной области переменной $Q^{2}$.

Мы, однако, используем другую возможность. Здесь по специальному соглашению для величин, подобных $D\left(Q^{2}\right)$-функции Адлера, представленных степенным рядом ТВ, процедура аналитизации применяется для каждой степени $\bar{\alpha}_{s}\left(Q^{2}\right)$ по отдельности. В результате возникает новое нестепенное разложение, в котором степени $\bar{\alpha}_{s}\left(Q^{2}\right)$ заменены новыми несингулярными функциями $A_{n}\left(Q^{2}\right)$. Такой алгоритм, впервые предложенный в работе [9], мы называем АТВ. Анализ амплитуд таких процессов, как $e^{+} e^{-}$-аннигиляция в адроны и инклюзивный $\tau$-распад, а также правил сумм неупругого лептон-адронного рассеяния, проведенный по этому алгоритму, показал, что помимо петлевой стабильности результаты АТВ обладают по сравнению с обычным подходом весьма слабой чувствительностью к выбору схемы перенормировок. Иными словами, трехпетлевой уровень АТВ практически обеспечивает как петлевое насыщение, так и схемную инвариантность рассмотренных физических величин на всем интервале изменения импульсной или энергетической переменной.

Создается впечатление, что учет дополнительной информации о корректных аналитических свойствах приводит к тому, что уже первые члены нестепенного ряда АТВ достаточно хорошо приближают его сумму. Здесь уместно провести аналогию с процедурой суммирования пертурбативных разложений с учетом дополнительной информа- 
ции о поведении дальних членов ряда ТВ [46]. При этом также оказалось, что в рамках такого подхода учет первых нескольких членов петлевого разложения дает для аппроксимируемой функции выражение, которое практически не изменяется при учете следующих поправок.

В этой работе мы рассмотрели также структурные функции неупругого лептон-адронного рассеяния, которые являются более сложными объектами, нежели двухточечные функции, связанные так или иначе с представлением Челлена-Лемана. Для таких функций общие принципы квантовой теории поля, такие как ковариантность, эрмитовость, спектральность и причинность отражены в интегральном представлении Йоста-Лемана-Дайсона. Для применения аналитического подхода к определению $Q^{2}$-эволюции оказались удобными моменты структурных функций $\mathcal{M}_{n}\left(Q^{2}\right)$, соответствуюшие специальной скейлинговой переменной (4.6). Именно эти моменты, а не бьеркеновские или нахтмановские, обладают простыми аналитическими свойствами по $Q^{2}$. В работе установлена связь новых аналитических моментов $\mathcal{M}_{n}\left(Q^{2}\right)$ и операторного разложения, в котором тензорная структура матричных элементов операторов по нуклонным состояниям должна быть фиксирована исходя из условия их ортогональности импульсу нуклона.

Благодарности. Нам приятно выразить благодарность В.С. Владимирову, А. В. Ефремову, Б. И. Завьялову, В. А. Мещерякову, С. В. Михайлову и О. В. Теряеву за полезное обсуждение полученных результатов. Мы также признательны РФФИ (гранты № 96-15-96030 и № 99-01-00091) и INTAS (грант № 96-0842) за поддержку.

\section{Список литературы}

[1] Н. Н. Боголюбов, Д. В. Ширков. ДАН СССР. 1955. Т. 103. С. 203; Р. 391.

[2] Н. Н. Боголюбов, Д. В. НІиков. ЖЭТФ. 1956. Т. 30. С. 77; N. N. Bogoliubov, D. V. Shirkov. Nuovo Cimento. 1956. V. 3. P. 845.

[3] Н. Н. Боголюбов, А. А. Логунов, Д. В. Ширков. ЖЭТФ. 1959. Т. 37. С. 805.

[4] H. Н. Боголюбов, В.С. Владимиров А.Н. Тавхелидзе. ТМФ. 1972. Т. 12. С. 3; Р. 305.

[5] Н. Н. Боголюбов, Д. В. Ширков. Введение в теорию квантованных полей. М.: Наука, 1973, 1976, 1986 (гл. “Дисперсионные соотношения").

[6] D. V. Shirkov, I. L. Solovtsov. Краткие сообщения ОИЯИ. 1996. ㄲo 2[76]-96. С. 5. hep-ph/9604363.

[7] D. V. Shirkov, I. L. Solovtsov. Phys. Rev. Lett. 1997. V. 79. P. 1209.

[8] I. L. Solovtsov, D. V. Shirkov. Phys. Lett. B. 1998. V. 442. P. 344.

[9] K. A. Milton, I. L. Solovtsov, O. P. Solovtsova. Phys. Lett. B. 1997. V. 415. P. 104.

[10] О. П. Соловцова. Письма в ЖЭТФ. 1996. Т. 64. С. 664.

[11] K. A. Milton, I. L. Solovtsov, O. P. Solovtsova. Analytic Perturbative Approach to QCD. Talk given at the XXIX Int. Conference on HEP. Vancouver, B. C., Canada, July 23-29, 1998 (to be published in the Proceed.); preprint OKHEP-98-06. Oklahoma Univ., 1998. hep-ph/9808457.

[12] Д. В. Ширков. ТМФ. 1999. Т. 119. С. 55; Renormalization group, causality, and nonpower perturbation expansion in QFT. Preprint E2-98-311. Dubna: JINR, 1998. hep-th/9810246.

[13] K. A. Milton, I. L. Solovtsov, O. P. Solovtsova. Phys. Lett. B. 1998. V. 439. P. 421.

[14] K. A. Milton, I. L. Solovtsov, O.P. Solovtsova. The Gross-Llewellyn Smith sum rule in the analytic approach to perturbative QCD. Preprint OKHEP-98-07. Oklahoma Univ., 1998. hep-ph/9809513.

[15] K. A. Milton, I. L. Solovtsov. Phys. Rev. D. 1997. V. 55. P. 5295.

[16] K. A. Milton, O. P. Solovtsova. Phys. Rev. D. 1998. V. 57. P. 5402.

[17] И. Ф. Гинзбург, Д. В. Ширков. ЖЭТФ. 1965. Т. 49. С. 335. 
[18] D. V. Shirkov. Nucl. Phys. B. 1990. V. 332. P. 425.

[19] D. V. Shirkov. Lett. Math. Phys. 1976. V. 1. P. 179.

[20] B. A. Magradze. The gluon propagator in analytic perturbation theory. Talk given at 10th Intern. Seminar on High-Energy Physics (Quarks 98). Suzdal, Russia, 18-24 May 1998. Preprint G-TMI-98-08-01, Tbilisi, 1998. hep-ph/9808247.

[21] E. Gardi, G. Grunberg, M. Karliner. Can the QCD running coupling have a causal analyticity structure? Preprint TAUP-2503-98. Paris, 1998. hep-ph/9806462.

[22] A. C. Mattingly, P. M. Stevenson. Phys. Rev. D. 1994. V. 49. P. 437.

[23] Yu. L. Dokshitzer, V. A. Khoze, S. I. Troyan. Phys. Rev. D. 1996. V. 53. P. 89.

[24] E. C. Poggio, H. R. Quinn, S. Weinberg. Phys. Rev. D. 1976. V. 13. P. 1958.

[25] P. M. Stevenson. Phys. Rev. D. 1981. V. 23. P. 2916.

[26] S. G. Gorishny, A. L. Kataev, S. A. Larin. Phys. Lett. B. 1991. V. 259. P. 144.

[27] F. Jegerlehner. Nucl. Phys. C (Proc. Suppl. ). 1996. V. 51. P. 131; Hadronic vacuum polarization contribution to $g-2$ of the leptons and $\alpha\left(M_{z}\right)$, hep-ph/9606484.

[28] S. Eidelman, F. Jegerlehner, A. L. Kataev, O. Veretin. Testing nonperturbative strong interaction effects via the Adler function. Preprint DESY 98-206. Hamburg, 1998. hep-ph/9812521.

[29] J. Chyla, A. L. Kataev, S. A. Larin. Phys. Lett. B. 1991. V. 261. P. 269.

[30] P. A. Raczka, A. Szymacha. Phys. Rev. D. 1996. V. 54. P. 3073.

[31] W. Celmaster, R. J. Gonsalves. Phys. Rev. D. 1979. V. 20. P. 1420.

[32] P. A. Raczka. Z. Phys. C. 1995. V. 65. P. 481.

[33] Review of Particle Physics. The European Phys. J. C. 1998. V. 3.

[34] E. Braaten. Phys. Rev. Lett. 1988. V. 60. P. 1606; Phys. Rev. D. 1989. V. 39. P. 1458.

[35] E. Braaten, S. Narison, A. Pich. Nucl. Phys. B. 1992. V. 373. P. 581.

[36] K. A. Milton, I. L. Solovtsov, V. I. Yasnov. Analytic perturbation theory and renormalization scheme dependence in $\tau$-decay. Preprint OKHEP-98-01. Oklahoma Univ., 1998. hep-ph/9802262.

[37] T. Coan et al. (CLEO Collaboration). Phys. Lett. B. 1996. V. 356. P. 580.

[38] R. Jost, H. Lehmann. Nuovo Cimento. 1957. V. 5. P. 1598.

[39] F. J. Dyson. Phys. Rev. 1958. V. 110. P. 1460.

[40] Н. Н. Боголюбов, А. А. Логунов, А. И. Оксак, И. Т. Тодоров. Общие принципы квантовой теории поля. М.: Наука, 1987.

[41] В. С. Владимиров, Ю.Н. Дрожжжинов, Б. И. Завьялов. Многомерные тауберовы теоремы для обобщенных функций. М.: Наука, 1986.

[42] O. Nachtmann. Nucl. Phys. B. 1973. V. 63. P. 237.

[43] B. Geyer, D. Robaschik, E. Wieczorek. Fortschr. Phys. 1979. V. 27. Р. 75; Б. Гайep, Д. Робашик, Э. Вищорек. ЭЧАЯ. 1980. Т. 11. С. 132.

[44] W. Wetzel. Nucl. Phys. B. 1978. V. 139. P. 170.

[45] S. Deser, W. Gilbert, E. C.S. Sudarshan. Phys. Rev. 1960. V. 117. P. 266.

[46] Д. И. Казаков, О. В. Тарасов, Д. В. ШІиков. ТМФ. 1979. Т. 38. С. 15; D. I. Kazakov, D. V. Shirkov. Fortschr. Phys. 1980. V. 28. P. 465. 